\title{
Der Schadensersatzanspruch aus Vertrag
}

\author{
System und Perspektiven
}

von Prof. Dr. Dr. Stefan Grundmann, LL.M., Erlangen-Nürnberg

Inhaltsübersicht

I. Überblick und Zielsetzung ....................... 570

1. Die Schwerpunkte der Schuldrechtsmodernisierung -

der Schadensersatz aus Vertrag als Paradigma ................ 571

2. Veränderte Aufgaben der Rechtswissenschaften

im Bürgerlichen Recht? ........................ 575

II. Untersuchungsgegenstand und Frage nach der Leitunterscheidung . . . . 576

1. Die untersuchten Regeln im Schadensersatzrecht . . . . . . . . . . 576

2. Systemfrage - die Frage nach der Leitunterscheidung . . . . . . . . . . 576

III. Das System des vertraglichen Schadensersatzes dem Grunde nach . . . . . . 577

1. Unterscheidung herkömmlich nach Verstoßtatbeständen . . . . . . . . . . 577

2. Vertraglicher Schadensersatz und Unmöglichkeit ............. 578

a) Schadensersatz statt der Leistung bei Unmöglichkeit (\283 BGB) . . 579

b) Schadensersatz bei anfänglich objektiver Unmöglichkeit

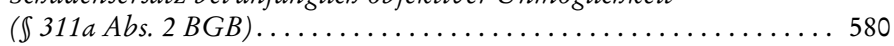

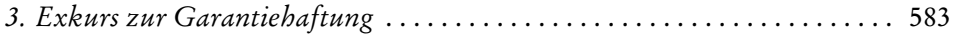

a) Der Aspekt des Kennenmüssens in $\int 311$ a Abs. 2 BGB

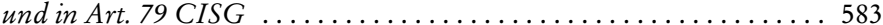

b) Garantiehaftung bei anfänglichem Unvermögen . . . . . . . . . . . 584

4. Vertraglicher Schadensersatz und Schlechterfüllung . . . . . . . . . . . . . 585

a) Belastungswirkung des Schadensersatzes statt der Leistung . . . . . . 586

b) Sonderbehandlung der Schlechterfüllung wäbrend des

Gesetzgebungsverfabrens ..................... 587

c) Sonderbehandlung in der verabschiedeten Fassung

zurückgedrängt $\left(\iint 281 \mathrm{f} . B G B\right) \ldots \ldots \ldots \ldots \ldots \ldots \ldots \ldots 8 \ldots \ldots \ldots$

5. Vertraglicher Schadensersatz und Verzug................ 590

a) Ein zweiter Schadensersatzanspruch mit Zusatzanforderungen . .... . 590

b) System weitgehend obne Zusatzanforderungen denkbar:

Fälligkeitszins bei Beachtlichkeit weitergehender Verzugsschäden . . 592

c) Auch realisiertes System mit nur schwachen Zusatzanforderungen . . . 593

6. Fazit: Rechtsfolgenorientierung statt Tatbestandsorientierung . . . . . . 594

7. Stellung im sonstigen Leistungsstörungsrecht . . . . . . . . . . . . . . . 595

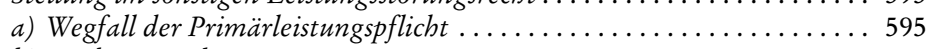

b) Rücktrittsrecht ............................. 596

IV. Nur eine kategoriale Änderung bei Inbalt und Höbe

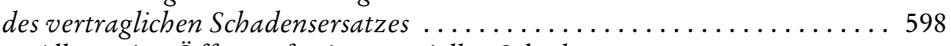

1. Allgemeine Öffnung für immateriellen Schadensersatz

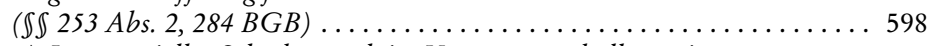

a) Immaterieller Schaden auch im Vertrags-und allgemein

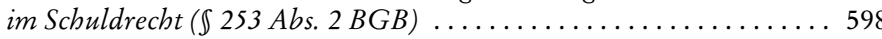

Archiv für die civilistische Praxis, Bd. 204 (2004), S. 569-605

(C) Mohr Siebeck - ISSN 0003-8997 
b) Ersatz immateriellen Schadens auch obne Verletzung der Person

des Anspruchsstellers (etwa $\int 284$ BGB).................. 599

2. Öffnung auch rechtsvergleichend und europarechtlich nabeliegend .....600

V. Resümee und Ausblick ................................. 602

Die Schuldrechtsmodernisierung war die tiefgreifendste Reform des BGB im Vermögensrecht seit 1900. System- und Kategorienbildung ist jetzt wichtig. Die großen „Systementdeckungen“ zum BGB wurden fast alle in den ersten gut zwei Jahrzehnten nach 1900 gemacht. Kategorienbildung ist ein Gleichheits- und Rechtssicherheitspostulat, in der Europäischen Diskussion aber auch conditio sine qua non, um neue Lösungen des BGB „transportieren“ zu können. Für den Schadensersatzanspruch aus Vertrag, wohl das Herzstück des neuen Leistungsstörungsrechts, wird im Folgenden argumentiert, dass die Differenzierung nach Verstoßtatbeständen praktisch keine Rolle mehr spielt, obwohl sie gesetzgebungstechnisch 2001 wieder „rehabilitiert“ (Canaris) wurde. Prägend ist vielmehr die Abschichtung in den Rechtsfolgen. Dies wird für Unmöglichkeit, Schlechterfüllung und Verzug durchgespielt. Darin wird der Übergang von einem Konzept der Freiheitsethik um 1900, in dem Verpflichtungen aus einzelnen „Verbots-“ oder Verstoßtatbeständen abgeleitet wurden und diese daher $\mathrm{zu}$ individualisieren waren, hin $\mathrm{zu}$ einem Recht für Märkte im Jahre 2000 gesehen, in dem unzählige, häufig anonyme Transaktionen zu bewältigen sind und daher das Resultat „Abweichung vom Plan des Vertrags“ ganz im Vordergrund steht. All dies hat auch erhebliche praktische Konsequenzen.

\section{I. Überblick und Zielsetzung}

So umstritten die Reform auch war, sie ist Wirklichkeit und gibt europaweit Anstöße, etwa in Italien und Österreich. ${ }^{1}$ Und das sind - neben Frankreich - die Mitgliedstaaten mit den großen alten Codices. Die Bedenkzeit ist länger als in Deutschland. ${ }^{2}$ Und hierzu kam es möglicherweise überhaupt nur, weil Deutschland vorangegangen war.

${ }^{1}$ Der Beitrag geht zurück auf einen Vortrag an der Humboldt-Universität am 18.2.2003, für Anregungen in der Diskussion danke ich herzlich. Überlegungen zu einer großen Reform auch etwa in Schaner/Fischer-Czermak (Hrsg.), Das ABGB auf dem Weg ins 3. Jahrtausend, 2003 (im österreichischen Justizministerium); für Italien vgl. den von Vita De Giorgi organisierten Kongress, La riforma dello Schuldrecht tedesco: un modello per il futuro diritto europeo delle obbligazioni e dei contratti? (3/ 2003, im Erscheinen) - inhaltlich gerade auch zum italienischen Recht.

${ }^{2}$ In Deutschland vielfach angemahnt, vgl. nur Ernst, Zum Fortgang der Schuld- 


\section{Die Schwerpunkte der Schuldrechtsmodernisierung - der Schadensersatz aus Vertrag als Paradigma}

Die Schuldrechtsmodernisierung hatte drei Reformschwerpunkte.

Den ersten - objektiv wichtigsten $-^{3}$ bildet die Kaufrechtsreform, bei der der BGB-Gesetzgeber jedoch durchweg gebunden war. In der Tat erscheinen $\int \mathbb{S} 433 \mathrm{ff}$. BGB weitestgehend als eine Kopie der Kaufrechts-Richtlinie, ${ }^{4}$ so dass jedenfalls für das Kaufrecht selbst grundsätzlich auf diese als das Original $\mathrm{zu}$ rekurrieren ist. ${ }^{5}$ Die großen rechtspolitischen Neuerungen beruhen hier auf Europäischer Vorgabe. Eine Frage von Gewicht war freilich autonom zu entscheiden, letztlich fiel die Entscheidung weitgehend im Konsens: ob das Europäische Kaufrecht nur für Verbraucherkäufe umzusetzen sei oder nicht besser als allgemeines Kauf- und Schuldrecht, wie das letztlich der deutsche Gesetzgeber annahm. ${ }^{6}$ Das ist in der rechtsvergleichenden Umschau keines-

rechtsreform, in: Ernst/Zimmermann (Hrsg.), Zivilrechtswissenschaft und Schuldrechtsreform - zum Diskussionsentwurf eines Schuldrechtsmodernisierungsgesetzes des Bundesministeriums der Justiz, 2001, 559, 603-605 et passim; relativierend: Canaris, Die Reform des Rechts der Leistungsstörungen, JZ 2001, 499, $523 \mathrm{f}$. Ähnlich für Europa: van Gerven, Codifying European Private Law - Top down and bottom up, in: Grundmann/Stuyck (Hrsg.), An Academic Greenpaper on European Contract Law, 2002, 405, 432: „Festina lente“.

3 Im Europäischen Vertragsrecht und im internationalen Einheitsrecht werden die jeweiligen Kaufrechtsakte in der Tat als die wichtigsten angesehen: Für die KaufrechtsRichtlinie (Fn. 4) konstatierte dies Emma Bonino selbst, die hierfür damals zuständige EU-Kommissarin; zitiert nach Trochu, Vente et garanties des biens de consommation directive CE no 1999-44 du 25 mai 1999, Dalloz 2000, Chron., 119, 119; entsprechend für das UN-Kaufrecht: Mitteilung der Kommission an den Rat und das Europäische Parlament zum Europäischen Vertragsrecht, $\mathrm{KOM}(2001) 398$ endg., S. 9.

${ }^{4}$ Richtlinie 1999/44/EG des Europäischen Parlaments und des Rates vom 25.5.1999 zu bestimmten Aspekten des Verbrauchsgüterkaufs und der Garantien für Verbrauchsgüter, ABl.EG 1999 L 171/12; Vorschlag vom 23.8.1996, KOM(95) 520 endg. = ABl.EG 1996 C 307/8; geänderter Vorschlag vom 7.4.1998, ABl.EG 1998 C 148/12; Stellungnahmen 1998 C 104/30 (Europäisches Parlament), 1997 C 66/5 (Wirtschafts- und Sozialausschuss); 1998 C 333/46 und 98/226 (Gemeinsamer Standpunkt und Beschluss des Europäischen Parlaments).

${ }^{5}$ Dazu Grundmann/Bianca (Hrsg.), EU-Kaufrechts-Richtlinie - Kommentar, 2002 (dort auch die deutsche und internationale Literatur zur Richtlinie); Übersichtsaufsätze zur Neufassung des deutschen Kaufrechts von Brüggemeier, Das neue Kaufrecht des Bürgerlichen Gesetzbuches, WM 2002, 1376-1393; Westermann, Das neue Kaufrecht, NJW 2002, 241-253; Schubel, Schuldrechtsmodernisierung 2001/2002 Das neue Kaufrecht, JuS 2002, 313-319.

${ }^{6}$ Dafür vor allem: früh Gass, Die Schulrechtsüberarbeitung nach der politischen Entscheidung, FS Rolland 1999, 129, 137-143 (eher implizit); sodann Brüggemeier, Zur Reform des deutschen Kaufrechts - Herausforderungen durch die EG-Verbrauchsgüterkaufrichtlinie, JZ 2000, 529, 529; Grundmann, Generalreferat: Internationalisierung und Reform des deutschen Kaufrechts, in: Grundmann/Medicus/Rolland (Hrsg.), Eu- 
wegs selbstverständlich. ${ }^{7}$ Das hat zur Folge, dass - trotz im Grundsatz verschiedener Standpunkte in dieser Frage - im konkreten Fall das Gebot richtlinienkonformer Auslegung sehr weitreichend gilt. Im konkreten Fall, im deutschen Kaufrecht, sind - soweit in denselben Normen geregelt - das Recht der Kaufverträge zwischen beruflich Tätigen und auch der durch die Richtlinie nicht geregelte Schadensersatz richtlinienkonform auszulegen. ${ }^{8}$

Den zweiten Schwerpunkt bildet die Integration des Verbraucherrechts. Materiell handelt es sich auch hier weitestgehend um Recht Europäischen Ursprungs. ${ }^{9}$ Die Frage der Integration ist etwas anders gelagert als beim Kaufrecht, denn Ziel war nicht oder nur in wenigen Fällen eine Ausweitung des persönlichen Anwendungsbereichs der jeweiligen Regeln. Dennoch betrifft auch die Frage der Reintegration das Verhältnis zwischen Verbraucherrecht und allgemeinem Privatrecht und dennoch ist der Standort - im Hauptgesetz des Privatrechts oder aber in Nebengesetzen und in Deutschland bis-

ropäisches Kaufgewährleistungsrecht - Reform und Internationalisierung des deutschen Schuldrechts, 2000, 281, 284-288; Westermann, Kaufrecht im Wandel, in: Schulzel Schulte-Nölke (Hrsg.), Die Schuldrechtsreform vor dem Hintergrund des Gemeinschaftsrechts, 2001, 109, 110; Reich, Die Umsetzung der Richtlinie 1999/44/EG in das deutsche Recht, NJW 1999, 2397, 2398 f. („Das Kaufrecht ist insgesamt neu zu justieren."); auch Gegner einer sog. „großen Lösung“ (mit Reform des allgemeinen Leistungsstörungsrechts), etwa Ernst/Gsell, Kaufrechtsrichtlinie und BGB - Gesetzentwurf für eine „kleine Lösung“ bei der Umsetzung der EU-Kaufrechtsrichtlinie, ZIP 2000, 1410, 1410. Grundsätzlich jetzt Drexl, Verbraucherrecht - Allgemeines Privatrecht Handelsrecht, in: Schlechtriem (Hrsg.), Wandlungen des Schuldrechts, 2002, 97, 105; Grundmann, Verbraucherrecht, Unternehmensrecht, Privatrecht - warum sind sich UN-Kaufrecht und EU-Kaufrechts-Richtlinie so ähnlich?, AcP 202 (2002), 40, 57-70.

7 Zur Umsetzung: Grundmann/Bianca (Fn. 5), jeweils Einl. Rn. 129 ff., und zwar deutsche Fassung: $\mathbb{S} \$ 433 \mathrm{ff}$. BGB und Österreich; englische Fassung: England und Niederlande; französische Fassung: Belgien und Frankreich; italienische Fassung: Italien und Spanien.

${ }^{8} \mathrm{Im}$ deutschen Kaufrecht geht nämlich auch der nationale Anwendungsbefehl wohl dahin, dass Konzepte wie das der Vertragswidrigkeit, Vertragsmäßigkeit als Primärleistungspflicht und angemessenen Frist etc.. obwohl nur teils der Umsetzung dienend, teils hingegen autonom verwandt, in allen Fällen stets gleich auszulegen seien: Grundmann, in: Grundmann/Bianca (Fn. 5), Einl. Rn. 26, dort auch zum Streit, ob das Gebot richtlinienkonformer Auslegung für die autonom gesetzten Normen, die auf dasselbe Konzept rekurrieren, im Europarecht oder im nationalen Recht fußt; siehe auch Bärenz, Die Auslegung der überschießenden Umsetzung von Richtlinien am Beispiel des Gesetzes zur Modernisierung des Schuldrechts, DB 2003, 375.

9 Kompaktkommentare: Grundmann, Europäisches Schuldvertragsrecht - das Europäische Recht der Unternehmensgeschäfte (nebst Texten und Materialien zur Rechtsangleichung), 1999 (2. Aufl. und englische Aufl. in Vorbereitung für 2004); Quigley, European Community Contract Law, 1997; für eine Reihe dieser Rechtsakte ausführlicher Grabitz/Hilf/Wolf (Hrsg.), Das Recht der Europäischen Union, Band III, 2002. 
her sogar noch verstreut - zumindest symbolträchtig. Er ist wohl sogar darüber hinaus auch von nicht unerheblicher Bedeutung für die Intensität der Diskussion zum jeweiligen Gebiet und der jeweiligen Regel sowie für das stetige Vergleichen und Prüfen von allgemeiner Privatrechtsregel und abweichender Sonderregel. ${ }^{10}$ Die Integration wurde weit überwiegend befürwortet, ${ }^{11}$ allerdings auch recht wenig von klassischen Bürgerlichrechtlern thematisiert.

Das „Schwergewicht“ der Reform liegt - privatrechtsdogmatisch - im Leistungsstörungsrecht - obwohl hier die rechtspolitischen Neuerungen ungleich geringer sind als im Kaufrecht, dort mit den Entscheidungen auf Europäischer Ebene. Das Leistungsstörungsrecht steht im Vordergrund aus der Perspektive der deutschen Vertragsrechtsdogmatik, vor allem jedoch in der allgemeinen Wahrnehmung: Nur in diesem Bereich wirkten die Ergebnisse der Schuldrechtskommission 1984-92 als Modell und standen zugleich auf dem Prüfstand. Nur zu diesem Bereich entzündete sich der überaus heftige Streit in der Wissenschaft. ${ }^{12}$ Für diesen Bereich tagte früh im Jahre 2001 eine hochrangig besetzte Kommission, bestehend fast nur aus Professoren, die, will man nicht einseitig einen Namen in deren Vordergrund rücken, als Leistungsstörungskommission (2001) anzusprechen ist. ${ }^{13}$ Deutlich gemischter

${ }^{10}$ Grundmann, AcP 202 (2002), 40, 69.

11 Dörner, Die Integration des Verbraucherrechts in das BGB, in: Schulze/SchulteNölke (Fn. 6), 177 f.; Grundmann (Fn. 6), 281, 284-288; Pfeiffer, Die Integration von "Nebengesetzen" in das BGB, in: Ernst/Zimmermann (Fn. 2), 481, 484-500 und 523 f.; Schmidt-Räntsch, Reintegration der Verbraucherschutzgesetze durch den Entwurf eines Schuldrechtsmodernisierungsgesetzes, in: Schulze/Schulte-Nölke (Fn. 6), 169-176; a. A. zur Integration des AGB-Gesetzes etwa Ulmer, Das AGB-Gesetz: ein eigenständiges Kodifikationswerk, JZ 2001, 491.

12 Bundesminister der Justiz (Hrsg.), Abschlußbericht der Kommission zur Überarbeitung des Schuldrechts, 1992. Zum Streit, d.h. vor allem zur Alternative "kleine“ oder "große Lösung" in der Schuldrechtsreform, statt vieler Ernst (Fn. 2); Lorenz/ Riehm, Lehrbuch zum neuen Schuldrecht, 2002, 2; deutlich erinnert man sich noch an die ganzseitigen Anzeigen in der FAZ mit einer Gegnerschaft von mehr als 200 Zivilrechtslehrern gegen die „große Lösung“. Für eine „kleine“ Lösung plädierten etwa Dauner-Lieb, Die geplante Schuldrechtsmodernisierung - Durchbruch oder Schnellschuß?, JZ 2001, 8, 15-18; Ernst, in: Ernst/Zimmermann (Fn. 2), 559, 598-605; Gsell, Kaufrechtsrichtlinie und Schuldrechtsmodernisierung, JZ 2001, 65, 75; Kohler, Das Rücktrittsrecht in der Reform, JZ 2001, 325, 336 f.; Lieb, Vom Beruf unserer Zeit zur Modernisierung des Schuldrechts, in: Ernst/Zimmermann (Fn. 2), 553-558; Wilhelm, Schuldrechtsreform 2001, JZ 2001, 861-869; Zimmermann, Schuldrechtsmodernisierung?, in: Ernst/Zimmermann (Fn. 2), 1, 19-22.

${ }_{13}$ Abdruck der Ergebnisse in: Canaris, JZ 2001, 499, 524. Canaris bereitete zentrale Entscheidungen vor (vgl. Fn. 27, 121, 165 und 182 im Aufsatz) und wurde denn auch für die außerordentlich einberufene Zivilrechtslehrertagung in Berlin März 2001 beauftragt, die Ergebnisse vorzustellen und zu verteidigen. Vgl. auch die Gesetzesdokumentation Canaris (Hrsg.), Schuldrechtsmodernisierung 2002, 2002. 
besetzt (aus Ministerialbürokratie, Praxis und Wissenschaft) war vorher die Schuldrechtskommission (1984-92). Die neuen Lehrbücher verwenden hierauf stets mehr als die Hälfte des Raumes, hier liegt mit dem Münchener Kommentar (nunmehr in der Bearbeitung von Ernst) ${ }^{14}$ auch schon ein erster Großkommentar vor. Das Schwergewicht der Reform liegt jedoch nicht nur aus deutscher Sicht im Leistungsstörungsrecht, sondern möglicherweise, wenn man in die Zukunft blickt, auch aus Europäischer, weil in diesem Bereich auch europaweit die Entscheidungen noch zu treffen sind.

Leistungsstörungsrecht betrifft nun vor allem drei Problemkomplexe: den Wegfall des Primäranspruches - hier nur exkursweise mit angesprochen - und zwei Sekundäransprüche. Denn auf zwei Sekundäransprüche oder -rechte konzentriert sich das neue Schuldrecht dezidierter als das alte: Rücktritt mit Rückabwicklung (primär mit dem Ziel einer Gleichstellung mit dem Zeitpunkt vorher, ohne Vertragsverhandlung) und Schadensersatz (er soll den Gewinn aus dem Vertrag, das mehr, das lucrum ex negotiatione, ${ }^{15}$ zumindest wertmäßig verbürgen). Beide wurden in der Diskussion sehr unterschiedlich aufgenommen. Im Rücktrittsrecht ging die gewichtigste Neuerung dahin, dass das Gestaltungsrecht heute flächendeckend verschuldensunabhängig gewährt wird - rechtspolitisch kaum angegriffen und auch vom Äquivalenzprinzip her zwingend. ${ }^{16}$ Und auch für die Rechtsfolgen liest man im Resümee von Hagers Regensburger Grundsatzreferat immerhin: „Das Anliegen der Novelle, den Rücktritt ungeachtet des ... Untergangs zuzulassen ..., ist zu begrüßen. “17 Liest man demgegenüber zum Schadensersatz - in Regensburg im Referat von Ulrich Huber - so klingen die Überschriften durchweg wie folgt: „Erste gesetzgeberische Fehlleistung“, „zweite Fehlleistung“, „dritte Fehlleistung “ etc., und inhaltlich ist die Diktion noch vernichtender. ${ }^{18}$ Diese Stellungnahme datiert zwar noch aus der Zeit, bevor die Leistungsstörungs-

${ }^{14}$ Rebmann/Säcker/Rixecker (Hrsg.), Münchener Kommentar, Band 2a, Schuldrecht, Allgemeiner Teil, 4. Aufl., 2003.

${ }^{15}$ Zur parallelen (schon römischrechtlichen) Unterscheidung zwischen damnum emergens und lucrum cessans etwa Zimmermann, The Law of Obligations - Roman Foundations of the Civilian Tradition, 1990, 827.

16 Die überragende Bedeutung dieser Entscheidung in der Schuldrechtsmodernisierung betont etwa auch Canaris, JZ 2001, 499, 509 und 522 (auch zur rechtsethischen Rechtfertigung); rechtspolitisch ebenfalls befürwortend etwa: Medicus, Schuldrecht I Allgemeiner Teil ${ }^{15}$, 2004, Rn. 502; wohl auch Schlechtriem, Schuldrecht Allgemeiner Teil ${ }^{5}, 2003$, Rn. 421 („Anlehnung an das CISG“); und vorher schon Entsprechendes aus dem Gedanken der Systemstimmigkeit her fordernd MünchKommBGB/Grundmann, Band 2, $\$ 276$ Rn. 48 (für die damalige lex lata).

17 Hager, Das geplante Recht des Rücktritts und des Widerrufs, in: Ernst/Zimmermann (Fn. 2), 429, 453.

${ }_{18}$ Huber, Das geplante Recht der Leistungsstörungen, in: Ernst/Zimmermann (Fn. 2), 31 (kürzere Fassung auch in: ZIP 2000, 2137 und 2273). 
kommission (2001) die Arbeit aufnahm. Doch auch danach, auf der Zivilrechtslehrertagung in Berlin, war hierzu die Kritik am heftigsten. Am Schadensersatzanspruch aus Vertrag entzündete sich der Disput wie an wohl keinem anderen Institut.

\section{Veränderte Aufgaben der Rechtswissenschaften im Bürgerlichen Recht?}

Zur Geschichte des BGB drängt sich auf, dass der Großteil der kategorialen „Erfindungen“ im ersten Jahrzehnt nach 1900 oder im ersten Jahrhundertviertel gemacht wurden: von Staubs $\mathrm{pVV}^{19}$ über die c.i.c., aufbauend zwar auf Iherings früheren Ausführungen, zum Durchbruch gelangt jedoch im Linoleumrollenfall von $1911,{ }^{20}$ weiter über den Vertrag mit Schutzwirkung gegenüber Dritten ${ }^{21}$ bis hin zum Wegfall der Geschäftsgrundlage. ${ }^{22}$ Hierher zählt dann überhaupt die stärkere Nutzung generalklauselartiger Tatbestände, die später zwar prominent in Frage gestellt wurde, ${ }^{23}$ letztlich jedoch eines der zentralen Vehikel für den Durchbruch der Interessen- und Wertungsjurisprudenz bildete. Und dieser selbst bildet ja ohnehin die wohl wichtigste Entwicklung zum BGB. Zwar sind die Punkte nach 1945 auch grundsätzlich fortentwickelt worden, etwa die c.i.c. zur Vertrauenshaftung durch Canaris ${ }^{24}$ oder - in der Nähe zur Lehre vom Wegfall der Geschäftsgrundlage - die Lehre von den Neuverhandlungspflichten. ${ }^{25}$ Das ändert jedoch nichts an der besonderen Stellung der Zeit recht unmittelbar nach 1900.

Ab 2002 wird vergleichbar wichtig wie der Durchbruch der Wertungsjurisprudenz voraussichtlich nur, dass mit dem BGB die Herausforderung, die Europäisierung und internationale Modelle bilden, angenommen werden

19 Staub, Die positiven Vertragsverletzungen, 1904.

20 Ihering, Culpa in contrahendo, 4 (1861) JherJb. 1; RGZ 78, 239.

21 Maßgeblich hier der sog. Klosterarztfall von 1915: RGZ 87, 289; auch 91, 21; 98, $210 ; 127,222$.

22 Vgl. nur die große Monographie von Oertmann, Die Geschäftsgrundlage,1921.

23 Berühmt Hedemann, Die Flucht in die Generalklauseln - eine Gefahr für Recht und Staat, 1933.

${ }^{24}$ Canaris, Die Vertrauenshaftung im deutschen Privatrecht, 1971; unter seinen Schülern dann Hager, Verkehrsschutz durch redlichen Erwerb, 1990; Singer, Selbstbestimmung und Verkehrsschutz im Recht der Willenserklärungen, 1995; (auch kritische) Beleuchtungen aus wirtschaftsrechtlicher Sicht etwa bei Assmann, Prospekthaftung als Haftung für die Verletzung kapitalmarktbezogener Informationsverkehrspflichten nach deutschem und US-amerikanischem Recht, 1985, 640-644; Hopt, Nichtvertragliche Haftung außerhalb von Schadens- und Bereicherungsausgleich - zur Theorie und Dogmatik des Berufsrechts und der Berufshaftung, AcP 183 (1983) 608, bes. 640-644.

25 Nelle, Neuverhandlungspflichten, 1993; Martinek, Die Lehre von den Neuverhandlungspflichten - Bestandsaufnahme, Kritik ... und Ablehung, AcP 198 (1998), $329-400$. 
muss. Jedenfalls aber steht die deutsche Privatrechtswissenschaft in einer Zeit, die nach Kategorienbildung verlangt. Lebt die heutige Zeit schneller, so bleibt vielleicht ein Jahrzehnt oder gar nur ein Jahrfünft. Das Europäische Zivilgesetzbuch erscheint heute nicht weniger wahrscheinlich als im Jahre 1900 der Umbruch zu einer Republik und ihrem Privatrecht. Kategorie- und Systembildung hat daher heute einerseits dieselbe - gleichheits- und rechtssicherheitsorientierte - Aufgabe wie 1900, weil sie natürlich auch hilft, Fallmaterial breiter zu ordnen. Kategorie- und Systembildung ist heute jedoch zusätzlich wichtig, weil die deutsche Privatrechtswissenschaft ohne sie auch mit reformiertem Kodex in der Europäischen Entwicklung kein Gehör findet. Klarheit und Kommunizierbarkeit sind hier conditio sine qua non.

\section{Untersuchungsgegenstand und Frage nach der Leitunterscheidung}

\section{Die untersuchten Regeln im Schadensersatzrecht}

Die neuen Regeln zum Schadensersatz aus Vertrag sind: (1) den Grund, die Voraussetzungen spezifizierend: $\int \$$ 276-283 BGB, vor allem $\int \$$ 280-283 BGB und $\int \mathbb{S} 311 \mathrm{a}$ Abs.2, $325 \mathrm{BGB}$; und (2) Inhalt und Höhe spezifizierend: $\$ \$ 249-253$ BGB, wohl gar bis $\$ 254$ oder $\$ 257$ BGB, jedenfalls aber auch $\$ 284$ BGB.

Im Folgenden werden vor allem die Voraussetzungen behandelt. Inhalt und Höhe haben sich 2002 sogar zwei Mal geändert: mit der Schuldrechtsmodernisierung (zum 1.1.2002), mit der $\$ 284$ BGB eingeführt wurde, dann nochmals (zum 1.8.2002) mit dem Zweiten Schadensrechtsänderungsgesetz, ${ }^{26}$ das $₫ 249$ Abs. 2 S. 2 BGB zu USt-Fragen einführte, ${ }^{27}$ vor allem jedoch $\ 253$ Abs. 2 BGB zum immateriellen Schaden. $\$ \$ 284$ und 253 Abs. 2 BGB bezeichnen den einzigen Punkt, in dem von systemrelevanten Umbrüchen bei Höhe und Inhalt des Schadensersatzanspruchs gesprochen werden kann.

\section{Systemfrage - die Frage nach der Leitunterscheidung}

Im Folgenden werden auch Einzelprobleme aufgegriffen, massiert etwa zu \311a Abs. 2 BGB. Vor allem jedoch geht es um Fragen der Veränderung der Gesamtkategorie Schadensersatzrecht durch die Reform und aus dieser heraus werden die Einzelpunkte bewertet. Als Referenzwerke in Fragen der Sy-

${ }^{26}$ Zweites Gesetz zur Änderung schadensersatzrechtlicher Vorschriften vom 19.7.2002, BGBl. 2002 I, 2674.

${ }_{27}$ Dazu etwa Wagner, Das Zweite Schadensersatzrechtsänderungsgesetz, NJW 2002, 2049, 2057-2059. 
stem- und Kategoriebildung ${ }^{28}$ werden die „Klassiker“ herangezogen, der Aussagegehalt ist hier am größten. Dabei mögen freilich - und ohne Anspruch auf Vollständigkeit - auch Erstauflagen schon solche bilden, etwa die Lehrbücher von Huber/Faust oder Lorenz/Riehm oder die Großkommentierung von Ernst. Gerade die genannten Autoren sind ja auch prononciert international ausgerichtet, gegenüber Modellen aus dem Internationalen und Europäischen (Einheits-)Recht potentiell besonders offen.

System bilden, heißt zuvörderst, das Leitunterscheidungskriterium oder die Leitunterscheidungskriterien zu klären. Für den Schadensersatz dem Grunde nach hat sich dieses tiefgreifend geändert, und dies kann auch die Gesetzesfassung nicht verdecken (III.). Dies gilt übrigens vergleichbar, wenn auch hier allenfalls en passant mitbesprochen, für den Wegfall der Primärleistungspflicht und weitestgehend auch den Rücktritt (vgl. unten III. unter 7.). Für Inhalt und Höhe des Schadensersatzanspruchs haben demgegenüber die zwei Reformen weniger grundsätzliche Änderung gebracht als die eine Reform für die Frage nach dem Grund. In einem Punkt freilich, bei der Ersatzfähigkeit immateriellen Schadens, könnte eine grundsätzliche Trendwende eingeleitet worden sein (IV.).

\section{Das System des vertraglichen Schadensersatzes dem Grunde nach}

\section{Unterscheidung herkömmlich nach Verstoßtatbeständen}

Medicus behandelt den (gesamten) Schadensersatzanspruch - dem Grunde nach - auch nach der Schuldrechtsreform unter der Überschrift „Unmöglichkeit“. ${ }^{29}$ Canaris macht die dahinter stehende Idee von den Leitkriterien explizit und spricht von „Rehabilitierung der Kategorie Unmöglichkeit“ ${ }^{30} \mathrm{Er}$

${ }^{28}$ Zum Systemdenken grundlegend Canaris, Systemdenken und Systembegriff in der Jurisprudenz ${ }^{2}$, 1983. Zum Systemgedanken im Europäischen Vertragsrecht grundlegend und bis in die Einzelheiten ausdifferenziert: Riesenbuber, System und Prinzipien des Europäischen Vertragsrechts, 2003; sowie auch (zum Vertrags- und Gesellschaftsrecht): Grundmann, Das Thema Systembildung im Europäischen Privatrecht Gesellschafts-, Arbeits- und Schuldvertragsrecht, in: Grundmann (Hrsg.), Systembildung und Systemlücken in Kerngebieten der Harmonisierung - Gesellschaftsrecht, Arbeitsrecht, Schuldvertragsrecht, 2000, 1; punktuell Tröger, Zum Systemdenken im europäischen Schuldvertragsrecht - Probleme der Rechtsangleichung durch Richtlinien am Beispiel der Verbrauchsgüterkauf-Richtlinie, ZEuP 2003, 525.

${ }^{29}$ Medicus Schuldrecht I - Allgemeiner Teil ${ }^{13}$, 2002, $\$ 33$ Die Unmöglichkeit der Leistung (unter IV.), kurz auch noch zu $\$ 280$ BGB in: $\$ 35$ Andere Pflichtverletzungen (unter III. Einzelheiten, dort 1.) Im Wesentlichen unverändert auch die Neuauflage (Fn. 16).

30 Canaris, JZ 2001, 499, 508; das gilt dann natürlich auch für die sonstigen herkömmlich unterschiedenen Verstoßkategorien (Verzug, pVV etc.). 
sieht denn auch - und viele mit ihm - den wohl wichtigsten Fortschritt der Leistungsstörungskommission (2001) gegenüber den Vorschlägen der Schuldrechtskommission (1984-92), die sich stärker an internationalen Vorbildern orientierte, in der Wiedereinführung oder neuerlichen Stärkung der Kategorie Unmöglichkeit. ${ }^{31}$ Huber/Faust sprechen zumindest von Zweispurigkeit (Unmöglichkeit/sonstige Verstoßtatbestände). ${ }^{32}$ Mit Ernst spricht sich jedoch einer der prominentesten Zweifler am Entwurf Schuldrechtskommission auch keineswegs umfassend positiv zur Aufgliederung aus. ${ }^{33}$

Dies kann verallgemeinert werden: Die herkömmlich unterschiedenen Verstoßtatbestände werden noch immer als prägend verstanden, jedenfalls wird ihre nicht unerhebliche Bedeutung auch für den Eintritt (unterschiedlicher) Rechtsfolgen nicht grundsätzlich in Zweifel gezogen. Ebendies soll jedoch im Folgenden geschehen.

\section{Vertraglicher Schadensersatz und Unmöglichkeit}

Die Unmöglichkeit betreffen drei Vorschriften: $\$ 275$ BGB, der nicht den Schadensersatz regelt, sondern den Fortbestand der Primärleistungsverpflichtung in Natur und den alle Rechte iErg. ähnlich kennen. Es gibt ganze Rechtsordnungen, die Primärleistungsansprüche grds. gar nicht vollstreckbar stellen (keine „specific performance“). ${ }^{34}$ Daneben finden sich die wichtigsten

31 Gerade der Wegfall der Kategorie der Unmöglichkeit als eigenständiger Leistungsstörungstatbestand nach den Vorschlägen der Schuldrechtskommission sah sich heftiger Kritik ausgesetzt, vgl. Huber, Die Pflichtverletzung als Grundtatbestand der Leistungsstörung im Diskussionsentwurf eines Schuldrechtsmodernisierungsgesetzes, ZIP 2000, 2273, 2278 f.; Canaris, Zur Bedeutung der Kategorie der „Unmöglichkeit“ für das Recht der Leistungsstörungen, in: Schulze/Schulte-Nölke (Fn. 6), 43 ff.; Wilhelm/Deeg, Nachträgliche Unmöglichkeit und nachträgliches Unvermögen, JZ 2001, $223,230 \mathrm{ff}$.

32 Huber/Faust, Schuldrechtsmodernisierung - Einführung in das neue Recht, 2002, 10-12; ähnlich Lorenz/Riehm (Fn. 12) 147 („Unmöglichkeit wieder ... eigenständiger Tatbestand des Leistungsstörungsrechts").

${ }^{33}$ MünchKommBGB/Ernst, Band 2a, Vor $\$ 275$ Rn. 15 („Ob diese Zweispurigkeit ... Gewinn ..., muss danach bezweifelt werden“). Freilich zweifelt auch Ernst die Zweispurigkeit als „Fakt“ nicht an.

34 Vgl. etwa Jones/Goodhart, Specific Performance ${ }^{2}, 1996$, bes. 24-127; rechtsvergleichend Caytas, Der unerfüllbare Vertrag, 1984; für das englische Recht Collins, The Law of Contract ${ }^{4}, 2003$, 427-433. Deswegen lässt Art. 28 CISG diese Frage - als eine von wenigen - unvereinheitlicht und verweist für die Frage der Durchsetzbarkeit des Primäranspruches auf das nationale Recht. Schon hier sei vorweggenommen, dass $\$ 275$ BGB keineswegs nur für die Unmöglichkeit gilt, sondern auch für die Schlechtleistung (unten bei Fn. 71). Und auch die Verzögerung ist mit angesprochen, wenn auch die zeitweise Unmöglichkeit mitbehandelt wird. Letztere ist in der Gesetzesfassung zwar nicht mehr explizit geregelt (anders noch der Entwurf der Leistungsstörungskommis- 
Unmöglichkeitsregeln in $\mathbb{S} 283$ und 311 a BGB, wobei $\$ 311$ a Abs. 1 BGB, (auch inhaltlich) anders als früher, ohnehin nur die Rechtsfolge von $\mathbb{2} 275$ BGB auch für die anfängliche (objektive) Unmöglichkeit bestätigt: Bestätigt wird, dass nicht der Vertrag unwirksam ist, sondern allenfalls die Primärleistungspflicht entfällt, dass also in puncto Wirksamkeitshindernisse auch für die Unmöglichkeit heute umfassend das allgemeine Regime gilt (Leistungsstörungen kein Wirksamkeitshindernis). Neben $\$ 275$ BGB betreffen also alle Unmöglichkeitsregeln - \283 und \311a Abs. 2 BGB - den Schadensersatz aus Vertrag. Mit ihrer Betrachtung wird daher bereits nahezu vollständig die Frage beantwortet, ob denn die Wiedereinführung der Kategorie „Unmöglichkeit“ durch die Leistungsstörungskommission (2001) nötig war und ob sie einen Fortschritt gegenüber der internationaler ausgerichteten Lösung der Schuldrechtskommission 1984-92 darstellt.

a) Schadensersatz statt der Leistung bei Unmöglichkeit (\283 BGB)

Die Antwort fällt leicht bei $\ 283$ BGB. Die Norm regelt den Schadensersatz statt der Leistung. Aus der Durchsicht der Verweise ergibt sich, dass $\$ 281$ BGB umfassend für anwendbar erklärt wird (für die Fragen Teilunmöglichkeit, Ausnahme der Unerheblichkeit der Pflichtverletzung und Anwendbarkeit des Rücktrittsrechts). Hiervon gibt es nur eine Ausnahme: Auf das Erfordernis der Fristsetzung wird verzichtet ( $\$ 281$ Abs. 1 S. 1 BGB) und daher sind Ausnahmen hiervon und Äquivalente ( $\$ 281$ Abs. 2 und 3 BGB) ebenfalls irrelevant. Auch muss die Befreiung von der Primärleistung $(\mathbb{} 281$ Abs. 4 BGB) nicht nochmals angeordnet werden, weil sie sich aus $\$ 275$ BGB ergibt. Die Ausgangsfrage ist daher dahingehend zuzuspitzen, ob $\$ 283$ BGB nötig war, damit in Fällen, in denen die Primärleistungspflicht nach $\$ 275$ BGB entfällt, ein Schadensersatzanspruch statt der Leistung auch ohne Fristsetzung unzweifelhaft eröffnet ist. Die Frage ist ohne weiteres zu verneinen: Erhebt der Schuldner die Einreden nach $₫ 275$ Abs. 2 und 3 BGB, so verweigert er damit ernsthaft und endgültig die Erfüllung (Ausnahme vom Fristsetzungserfordernis nach $\mathbb{2} 281$ Abs. 21 . Alt. BGB). Beweist der Gläubiger, dass die Leistung unmöglich ist ( $\$ 275$ Abs. 1 BGB) - und unstreitig kann er sich nur unter dieser Voraussetzung auf $\$ 283$ BGB stützen,$-{ }^{35}$ überwiegt sein Gläubigerinteresse offensichtlich ein - eben völlig inexistentes - Schuldnerinteresse daran, dass er noch eine Frist und damit zweite Erfüllungschance er-

sion [2001]), doch weiterhin natürlich mit angesprochen und nur der Entwicklung in Dogmatik und Rechtsprechung überlassen: BT-Drucks. 14/7052, 183; Canaris, JZ 2001, 499, 508 und 516 (dort auch zum Entwurf der Kommission); Kropholler, Studienkommentar $\mathrm{BGB}^{6}, \$ 275 \mathrm{Rn} .1$.

${ }^{35}$ Vgl. nur MünchKommBGB/Ernst, $\$ 283$ Rn. 24 f. 
hält (Ausnahme vom Fristsetzungserfordernis nach $\$ 281$ Abs. 2, 2. Alt. BGB). $\$ 281$ Abs. 2 BGB würde also die Fälle, die $\$ 283$ BGB regelt, vollständig, unzweifelhaft und mit identischer Folge erfassen. Durch Ausbildung einer gesonderten Regelung wird unnötig der Anschein erweckt, Tatbestände oder Rechtsfolgen divergierten. Allenfalls ließe sich in Abs. 2 an eine (auch schon recht gekünstelt wirkende!) Klarstellung dergestalt denken, dass ein weiterer Satz angefügt wird: „Solch eine Weigerung ist darin zu sehen, dass

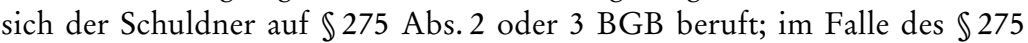
Abs. 1 BGB hat dieser kein Interesse an einer Fristsetzung." Die erste Unmöglichkeitssonderregel zum Schadensersatz ist demnach nur formal eine solche und daher überflüssig. Unmöglichkeit folgt dem allgemeinen Regime.

b) Schadensersatz bei anfänglich objektiver Unmöglichkeit

( $\int 311 a$ Abs. 2 BGB)

Komplexer ist $\ 311$ a BGB. Mit Abs. 1 wurde die Sondervorschrift in $\$ 306$ BGB a.F. aufgehoben, in der die anfängliche Unmöglichkeit als Wirksamkeitshindernis ausgestaltet war. Diese Sondervorschrift galt allgemein und zu Recht als überholt. Grundlegend war wieder ein „kategoriales“ Werk aus dem ersten Jahrzehnt, ${ }^{36}$ das wie manch anderes auch damals schon auf Rechtsvergleichung gestützt war. Wirksamkeitshindernisse ergeben sich aus keiner Form der Unmöglichkeit mehr, die systemfremde Ausnahme entfiel. Für den Bestand des Vertrages gelten also keine Sonderregeln für den Fall der Unmöglichkeit mehr, diese wurde dem allgemeinen Regime unterstellt. Der Vertrag ist freilich, da selbstverständlich $\$ 275$ BGB eingreift und die Primärleistungspflicht auch bei anfänglicher Unmöglichkeit nicht besteht, ${ }^{37}$ allein Grundlage für Sekundäransprüche.

Einen Sekundäranspruch regelt dann $₫ 311$ a Abs. 2 BGB. Mit dieser Norm wird der Bezugspunkt des Vertretenmüssens anders festgesetzt als üblich: Nach $\$ \$ 276,280$ BGB ist maßgeblich, ob der Schuldner das die Unmöglichkeit verursachende Ereignis zu vertreten hat, nach $\$ 311$ a Abs. 2 BGB hingegen, ob er seine Unkenntnis von der Unmöglichkeit - auch extern verursachter-vertreten musste. Die Überlegung und Aufdeckung dieses Zurechnungs-

${ }^{36}$ Rabel, Unmöglichkeit der Leistung, FS Bekker 1907, 171 = Gesammelte Aufsätze Bd. 1, 1965, 1. Aus der späteren, weitgehend einhelligen Kritik etwa Bundesminister der Justiz (Fn. 12), 145 (,allgemein als unsachgemäß angesehen“); MünchKommBGB/ Emmerich bzw. Thode, Band 2, Vor \$275 Rn. 4 f. bzw. \$306 Rn. 3.

37 Canaris, JZ 2001, 499, 500; Kropholler (Fn. 34), \$283 Rn. 1; MünchKommBGB/ Ernst, $\$ 275$ Rn. 18. Dazu, dass auch die Rechtsfolge des $\$ 275$ BGB nicht nur im Falle der Unmöglichkeit greift, unten 7. 
gesichtspunkts überzeugt durchaus. ${ }^{38}$ Freilich wird die Konsequenz im Systematischen nicht mehr gezogen. Es handelt sich nicht mehr um eine Sonderregel zum Vertragsschluss. Das war sie, solange die anfängliche Unmöglichkeit Wirksamkeitshindernis war, und nur so lange war die Regelung unter den Vertragsschlussregeln richtig angesiedelt $\left(\mathbb{S} \$ 305 \mathrm{ff}\right.$. BGB a.F., heute $\mathbb{\int} 311 \mathrm{ff}$. BGB). Heute handelt es sich um eine Sonderregel zum Vertretenmüssen. Die Streichung einer systemfremden Sondervorschrift in Verbindung mit einem Hinweis in der Bundestags-Drucksache hätte anstelle von $\$ 311 \mathrm{a}$ Abs. $1 \mathrm{BGB}$ für die kurze Eingewöhnungszeit wohl genügt. Systematisch konsequent für die wirklich wichtige Regelung - in Abs. 2 - wäre eine Stellung im Fragenkomplex Vertretenmüssen, in $\$ 276$ oder $\$ 280$ Abs. 1 BGB, als Spezialregel zum Bezugspunkt beim Vertretenmüssen. ${ }^{39}$ Dagegen lässt sich auch nicht einwenden, dass $₫ 311$ a BGB als ein Spezialfall der Haftung für eine vorvertragliche Pflichtverletzung im Anschluss an $₫ 311$ Abs. 2 BGB richtig platziert ist. Denn $\ 311$ Abs. 2 BGB definiert lediglich die Voraussetzungen, unter denen ein vorvertragliches Schuldverhältnis entsteht, fungiert jedoch im Gegensatz $\mathrm{zu} \ 311 \mathrm{a}$ Abs. 2 BGB nicht als eigenständige Anspruchsgrundlage.

Bei einer Verortung der Haftung für anfängliche Unmöglichkeit in $\$ 276$ oder $\$ 280$ Abs. 1 BGB wären eventuell auch die systematisch wie praktisch noch wichtigeren Folgefragen aufgefallen. Der Gedanke von $\$ 311$ a Abs. 2 BGB ist nämlich ein allgemeiner, der weder den anderen Bezugspunkt beim Vertretenmüssen verdrängt noch auf die Unmöglichkeit oder auf die anfängliche Leistungsstörung beschränkt ist. Im Einzelnen:

Zunächst ist zu fragen, ob der Schuldner bei anfänglicher Unmöglichkeit nur die verschuldete Unkenntnis oder Kenntnis vertreten muss oder auch die vorvertraglich verschuldete Unmöglichkeit, mit anderen Worten: ob $\mathbb{3} 11 \mathrm{a}$ Abs. 2 BGB $\$ 280$ BGB verdrängt ${ }^{40}$ oder neben ihn tritt, d.h. diese zweite

${ }^{38}$ Im Entwurf der Schuldrechtskommission war zwar $\$ 306$ BGB a.F. gestrichen worden, jedoch weder betont worden, dass (fortan) der Vertrag trotz anfänglicher objektiver Unmöglichkeit als wirksam zu behandeln sein sollte (jetzt $\$ 311$ a Abs. 1 BGB), noch auf den hier vorrangig einschlägigen Zurechnungsgesichtspunkt hingewiesen worden (jetzt $\$ 311$ a Abs. 2 BGB). Das Zweite wurde im Gesetzgebungsverfahren dann intensiv betont. Vgl. etwa MünchKommBGB/Ernst $\$ 311$ a Rn. 4, 15, 42; Palandt/ Heinrichs, \$311a Rn. 2; Lorenz/Riehm (Fn.12) 166.

39 Teils wird hier die Frage im Recht der Willensmängel verortet, die Anwendung von dessen Rechtsfolgen dann jedoch abgelehnt: Canaris, JZ 2001, 499, 507 f. (keine Anfechtbarkeit, allerdings eine analoge Anwendung von $\$ 122$ BGB erwägend). Die Nichtanwendung der Rechtsfolgen zeigt jedoch deutlich genug, dass es vorrangig um die Zurechnung von Leistungsstörungen („Vertretenmüssen“) geht.

40 So Canaris, JZ 2001, 499, 507; MünchKommBGB/Ernst \$311a Rn. 45; und offenbar Huber/Faust (Fn. 30), 213; Kropholler (Fn. 32), \$283 Rn.1, \311a Rn.3; Medicus (Fn. 16), 229 f. (Leistungspflicht hat nie bestanden); auch BT-Drucks. 14/6040, S. 165 . 
Haftungsbegründungsnorm unberührt lässt. Meist ist dem Schuldner in Fällen, in dem er das die Unmöglichkeit verursachende Ereignis zu vertreten hat, etwa weil seiner Organisation Verschulden vorzuwerfen ist, auch die Unkenntnis hiervon vorzuwerfen, jedoch nicht notwendig. Ein Frankfurter Freund, Weinliebhaber, hatte eine Partie Beau-Séjour Bécot subskribiert, hier, wie bei manchen Spitzenweinen, über einen weingutseigenen Vertriebskanal. Nicht alle Verträge konnten bedient werden, weil ein gefeuerter Küfer noch viele Regale umgestoßen hatte, hier wohl tatsächlich erst in der berühmten Nacht zuvor. Man mag argumentieren, solche Schäden müssten innerhalb von Stunden im Verkaufsgeschäft bekannt sein, wie jedoch, wenn Kontrollgänge häufiger als einmal täglich die Weinqualität gefährden könnten? Ab einem gewissen Punkt würde die Kategorie des Kennenmüssens gesprengt. Die gegenteilige Argumentation ginge dahin, dass heute in der professionellen Produktion auf Verkauf an den Kunden produziert wird und daher - wenn schon nicht allgemein unabhängig von Verschulden - zumindest auch Fehlverhalten in diesem Prozess - unabhängig von Kennenmüssen beim letzten Absatzglied - zuzurechnen ist. Der wesentliche Vorwurf ist dann eben doch der, dass die Unmöglichkeit selbst vorwerfbar verursacht wurde. $\$ 311$ a Abs. 2 BGB tritt also neben die Zurechnungsregeln in $\$ \$ 276,280$ Abs. 1 BGB und verdrängt sie nicht. Praktisch sehr wichtig ist all dies zugegebenermaßen noch nicht.

Zwei Ausweitungsfragen schließen sich an, potentiell weitreichende Implikationen scheint vor allem die Zweite zu haben. Zunächst ist zu fragen, warum der Zurechnungsgesichtspunkt nicht auch bei Schlechterfüllung gelten soll, wenn eine solche schon vor Vertragsschluss nicht mehr zu vermeiden ist. Soll Kennenmüssen irrelevant sein, wenn im Beispielsfall vor Vertragsschluss etwa durch Erdrutsch für kurze Zeit Wärme in die Kellerhallen gedrungen sei und dem Bouquet etwas schadete, dies der Vertriebsstelle bekannt hätte sein müssen, ein Feinschmeckerrestaurant den Wein ausschenkt und wichtige Stammkunden daraus einen casus belli machen? Der zweite Bezugspunkt für das Vertretenmüssen ist zwar nur für die anfängliche Unmöglichkeit formuliert worden: Zugerechnet wird neben Fällen, in denen der Schuldner die Leistungsstörung hätte beherrschen können ( $\$ 276$ BGB), auch die vorwerfbare Unkenntnis von Einflüssen, die der Schuldner nicht beherrschen konnte. Offensichtlich kann dieser Gedanke jedoch für jede Pflichtverletzung fruchtbar gemacht werden. Es ist nicht zwingend - und sogar ein wenig willkürlich -, die anfängliche Unmöglichkeit vollständig korrekter Erfüllung (anfänglich feststehende Schlechterfüllung) anders zu behandeln. In der Tat ist die Regelung in $\$ 276$ BGB offen genug, um solch eine Ausweitung fassen zu können. Die zweite Ausweitung ist noch radikaler: 


\section{Exkurs zur Garantiehaftung}

a) Der Aspekt des Kennenmüssens in $\int 311 a$ Abs. 2 BGB und in Art. 79 CISG

Es ist ebenfalls wenig plausibel, dass der gleiche Vorwurf - zwar war die Unmöglichkeit nicht beherrschbar, der Schuldner hätte aber von der Gefahr wissen müssen - nicht auch für nachträglich auftretende, aber schon vorher erkennbare Leistungshindernisse gelten soll. In der Tat diente $\$ 311 \mathrm{a}$ BGB ja vor allem dem Ziel, jede unnötige Ungleichbehandlung von anfänglichen und nachträglichen Leistungshindernissen (gesprochen wurde nur von Unmöglichkeit) zu beseitigen. ${ }^{41}$ Dieses Gesetzgeberziel fortgedacht, legt es nahe, dass in der Tat auch die nachträgliche Unmöglichkeit (und allgemeiner Leistungsstörung) nach $\ 311$ a Abs. 2 BGB zugerechnet werden kann: Ist nicht dem häufig Kränkelnden sein Wissen hiervon und auch Kennenmüssen vorzuwerfen, wenn er sich für ein erkennbar wichtiges Einmalereignis als unersetzbare Zentralperson verpflichtet - wenn auch nicht, dass er nach Vertragsschluss dann in der Tat krank wird? ${ }^{42}$

Dies freilich wäre ein bedeutsamer Schritt. Er wäre doch gleichbedeutend mit der Aussage, dass der Schuldner ein Leistungshindernis auch zu vertreten hat, wenn dieses nicht vollkommen unvorhersehbar war („Kennenmüssen“) und dies bei anfänglicher und nachträglicher Leistungsstörung, bei Unmöglichkeit und anderer Leistungsstörung. Mit anderen Worten: Der Schuldner wäre an sein Versprechen gebunden, es sei denn ein Leistungshindernis war nicht nur für ihn nicht beherrschbar, sondern auch nicht vorhersehbar. Mit Anerkennung des zweiten Bezugspunkts entfällt der eigentliche Unterschied zwischen dem traditionellen Verständnis der Verschuldenshaftung (bei objektiviertem Sorgfaltsbegriff, entsprechend $\$ 276$ BGB) und Art. 79 CISG, der als Paradebeispiel einer Garantiehaftung verstanden wird, die nur bei sog. höherer Gewalt eingeschränkt ist. Denn dort wird unterschieden zwischen den Hindernissen, die der Schuldner beherrschen konnte (hier greift bereits $\$ 276$ BGB), und den von außen hinzutretenden Hindernissen, die der Schuldner nicht beherrschen kann, die ihm aber nur in den Fällen nicht zuge-

${ }^{41}$ Bundesminister der Justiz (Fn. 12), 145 f.; Canaris, JZ 2001, 499, 507; BT-Drucks. 14/6040, S. 165 („Das entspricht dem Bestreben, die anfängliche Unmöglichkeit hinsichtlich der Rechtsfolgen genauso zu behandeln wie die nachträgliche“).

42 Bei der Erkrankung selbst handelt es sich nach der gesetzlichen Wertung wohl in der Tat um - nicht zu vertretende - Unmöglichkeit: Canaris, JZ 2001, 499, 504; MünchKommBGB/Ernst $\$ 275$ Rn. 39 (teils $\$ 275$ Abs. 1, teils Abs. 3 BGB); Palandt/Heinrichs, $\$ 275 \mathrm{Rn} .30$. 
rechnet werden, in denen sie für ihn auch nicht vorhersehbar waren. ${ }^{43}$ Letzteres würde auch $\$ 311 \mathrm{a}$ Abs. $2 \mathrm{BGB}$ statuieren, wenn er in der Tat allgemein zu verstehen wäre und der Gesetzgeberwille ernst genommen würde, keine Regelungsunterschiede zwischen anfänglichen und nachträglichen Leistungsstörungen mehr zuzulassen, wenn die fragliche Regelung von ihrem Regelungsziel her auf beide vergleichbar zutrifft.

Zwar wird die „höhere rechtsethische Überzeugungskraft“ des Verschuldensprinzips behauptet, ${ }^{44}$ die hier vorgetragene Überlegung spricht jedoch, wenn der $₫ 311$ a Abs. 2 BGB zugrunde liegende Gedanke überhaupt tragfähig ist, eher für eine Garantiehaftung in Form des Art. 79 CISG - ganz abgesehen davon, dass es Kollegen des Common Law stets schwer zu erklären ist, warum der Versprechensempfänger eher dazu prädestiniert sein soll, bei nicht vollständiger Erfüllung eines vertraglichen Versprechens den Schaden zu tragen als der Versprechende, und - etwas emotionaler - das, was sie gewöhnt sind zu denken, „rechtsethisch“ niedriger einzustufen ist. ${ }^{45}$

Der Regelungsgedanke des $₫ 311$ a Abs. 2 BGB ist also ein allgemeiner. Die Norm steht (1) am falschen Ort, verdrängt (2) nicht das Vertretenmüssen der Unmöglichkeit selbst (Frankfurter Weinliebhaber) und ist (3) keine Sonderregel, sondern zu verallgemeinern, und zwar auf die Fälle von Schlechterfüllung (Feinschmeckerlokal) und auf nachträgliche Leistungshindernisse (kränkelnder Alleinunterhalter), was aber dem Schritt zu einer nur bei höherer Gewalt eingeschränkten Garantiehaftung zumindest sehr nahe kommt.

\section{b) Garantiehaftung bei anfänglichem Unvermögen}

Das Gesagte wirkt sich auf den letzten verbleibenden Aspekt aus, der bei der Neuregelung des Problemkomplexes anfängliche Leistungshindernisse intensiv diskutiert wurde. Bekanntlich sprach sich vor der Reform die h.M. dafür aus, anfängliches Unvermögen dem Schuldner ohne Verschulden zuzurechnen (Garantiehaftung) und sollte eben dies zumindest nicht fortgeschrieben werden. ${ }^{46}$ Dass Unvermögen und objektive Unmöglichkeit für die Frage, ob der Primärleistungsanspruch entfällt, nicht (mehr) unterschiedlich behan-

${ }^{43} \mathrm{Zu}$ dieser Auslegung von Art. 79 CISG: Standinger/Magnus, Art. 79 CISG Rn. 7; Scblechtriem/Stoll, Art. 79 CISG Rn. 6-9.

${ }^{44}$ Etwa BT-Drucks. 14/6040, 165; Canaris, JZ 2001, 499, 506.

45 Den wohl berühmtesten Fall eines kontinentaleuropäischen Juristen, der diesen „Kultursprung“ tat und verinnerlichte, hat man in Rabel zu sehen. Vgl. zu dessen Plädoyer für eine (natürlich dispositive) Garantiehaftung im Vertrag: Rabel, Das Recht des Warenkaufs - eine rechtsvergleichende Darstellung, 2 Bände, 1958, 56-58 et passim.

${ }^{46}$ Canaris, JZ 2001, 499, 506; Lorenz/Riehm (Fn. 12) 168 f.; MünchKommBGB/ Ernst $₫ 311$ a Rn. 12. Für solch eine Garantiehaftung zuletzt noch BGHZ 144, 118, $120 \mathrm{f}$. 
delt werden sollten, steht (zu Recht) außer Streit. Die Diskussion betrifft also allein den Sekundäranspruch, konkret: den Schadensersatzanspruch. Hier ist die Gesetzgebungsgeschichte dahingehend zu interpretieren, dass sich die Befürworter der bisherigen Mehrheitsmeinung nicht in dem Sinne durchsetzen konnten, dass diese gesetzlich festgeschrieben wurde, eher im Gegenteil. ${ }^{47}$

Befürwortet man generell eine Garantiehaftung im Vertragsrecht, so konnte man in der bisher mehrheitlich vertretenen Garantiehaftung bei anfänglichem Unvermögen einen wichtigen Beispielsfall auch im deutschen Vertragsrecht für diese Lösung sehen, die ohnehin im Einheits- und vor allem auch im Gemeinschaftsrecht vordringt. ${ }^{48}$ Versteht man, stärker am jüngsten Gesetzgebungsakt orientiert, $\mathbb{\$} 311$ a Abs. 2 BGB in dem hier vorgetragenen Sinne, so kommt man ebenfalls nicht umhin, das anfängliche Unvermögen zumindest in der praktischen Anwendung besonders nahe bei der Garantiehaftung zu sehen: Das bereits vorliegende Leistungshindernis, das zudem für den Schuldner eigentümlich ist, muss sicherlich häufiger gekannt werden als die Gefahr eines zukünftigen Eintritts oder das externe, bereits bestehende Leistungshindernis. Formal wurde also die bisherige Mehrheitsmeinung nicht festgeschrieben, praktisch wird es jedoch den absoluten Ausnahmefall bilden, dass Kennenmüssen nicht angenommen wird und exakt der Vorwurf erhoben wird, der nach Art. 79 CISG relevant ist: Zwar mag das Leistungshindernis selbst für den Schuldner nicht beherrschbar gewesen sein, er hätte es jedoch kennen müssen.

\section{Vertraglicher Schadensersatz und Schlechterfüllung}

Unmöglichkeit ist also unzweifelhaft irrelevant für die Frage nach dem Schadensersatz statt der Leistung ( $\$ 283$ BGB) und für den Bestand des Vertrages (ausdrücklich $\ 311$ a Abs. $1 \mathrm{BGB}$ ), richtigerweise auch für den Wegfall der Primärleistungspflicht ( $\$ 275$ BGB, unten 7.) und für die Eröffnung einer zweiten Ebene des Vertretenmüssens (Kennenmüssen eines von außen herangetragenen, unbeherrschbaren Hindernisses). Wenn eine Kategorie irrelevant ist, erscheint es angezeigt, sie eher nur als Beispiel in der Hauptnorm aufzuzählen als durch Ausbildung eigener Normen den Anschein zu erwecken, Rechtsfolgen seien für bestimmte Verstoßkategorien andere.

Für einen zweiten Verstoßtatbestand schließt sich die parallele Frage an danach, ob denn wenigstens Schlechtleistung (pVV) als besondere Verstoßka-

47 BT-Drucks. 14/6040, 165. Kritisch dazu insbesondere U. Huber, Leistungsstörungen, Bd. I, 1999, S. 539; ders., Die Unmöglichkeit der Leistung im Diskussionsentwurf eines Schuldrechtsmodernisierungsgesetzes, ZIP 2000, 2137, 2149.

48 So MünchKommBGB/Grundmann, Band 2, \$276 Rn. 29, 31-33. 
tegorie von Bedeutung ist. $\$ 280 \mathrm{BGB}$, der den Schadensersatz neben der Leistung regelt, gilt offenbar allgemein für jeden vertraglichen Pflichtverstoß, nur für den Verzug (unten 5.) verweist $\$ 280$ Abs. 2 BGB auf Sondervoraussetzungen. Die genannte Frage stellt sich also nur für den Schadensersatz statt der Leistung. Hier gilt es, $\int \mathbb{S} 281$ und 282 BGB voneinander abzugrenzen, vorher jedoch die Gesetzgebungsgeschichte kurz zu betrachten und vor allem das Fristsetzungserfordernis und mit ihm das Modell kurz zu erläutern.

\section{a) Belastungswirkung des Schadensersatzes statt der Leistung}

Vom Fristsetzungserfordernis, dem zentralen Zusatzerfordernis ist auszugehen: Schadensersatz statt der Leistung ist vor allem aus zwei Gründen im typischen Fall belastender als Schadensersatz neben der Leistung: Der Leistende erhält seine Leistung zurück - häufig gilt sie nun als gebraucht, etwa das einmal zugelassene Kfz, oder ist nicht mehr gebrauchbar, etwa das etwas lückenhaft erstattete Individualgutachten. Die Leistung hat also häufig allein schon durch Erbringung (noch ohne Nutzung und damit auch ohne Nutzungsentgelt) erheblich an Wert eingebüßt, $20 \%$, manchmal $100 \%$. Und weil er dem Vertragspartner dessen vollen Schaden zu ersetzen hat, etwa einem Kunden den Betrag, den dieser zum Ersatzkauf benötigt, verliert der Leistende zudem seinen Geschäftsgewinn, häufig ca. $30 \%$ des Preises. ${ }^{49}$ Das hat der Schadensersatz statt der Leistung mit dem Rücktritt gemein, weshalb im Ergebnis beide gleiche Voraussetzungen haben (außer Vertretenmüssen, vgl. unten 7.). Daher gibt man dem schlecht Leistenden - zu Recht - eine zweite Chance (Fristsetzung), und gilt die Rechtsfolge nicht bei völlig unerheblichen Verstößen. All dies entspricht dem Modell des UN-Kaufrechts und der Kaufrechts-Richtlinie, obwohl diese bekanntlich den Schadensersatz nicht regelt. Dass diese Überlegung in der Schadensersatzregelung der European Principles nicht reflektiert ist (vgl. Art. 9-501 PECL), ${ }^{50}$ ist ein erheblicher Nachteil (im Vergleich zum deutschen Recht). Dort findet sich das Fristsetzungser-

${ }^{49} \mathrm{Zu}$ diesen beiden Nachteilen: Gómez, in: Grundmann/Bianca (Fn. 5), Einl. Rn. 108; Grundmann, AcP 202 (2002), 40, 50; zu Kostengesichtspunkten auch Wolf, Reform des Kaufrechts durch EG-Richtlinie - ein Vorteil für die Wirtschaft? Die möglichen Auswirkungen einer Richtlinie über den Verbrauchsgüterkauf und -garantien auf das deutsche Kaufrecht und die Wirtschaft, RIW 1997, 899, 902; und etwas verschlüsselt auch schon $\mathrm{KOM}(95) 520$ endg., 14; vgl. auch Bundesminister der Justiz (Fn. 12), 212 f.; Schlechtriem, Das geplante Gewährleistungsrecht im Licht der europäischen Richtlinie zum Verbrauchsgüterkauf, in: Ernst/Zimmermann (Fn. 2), 205, $218 \mathrm{f}$.

50 Lando/Beale (Hrsg.), Principles of European Contract Law, Part I and II, 2000, Part III 2003, 1. Teil schon 1996; in Deutsch: v. Bar/Zimmermann für die Kommission für Europäisches Vertragsrecht, Grundregeln des Europäischen Vertragsrechts., Teile I und II, 2002. 
fordernis nur in der Rücktrittsregelung und auch (etwas zu pauschal) nicht bei erheblichem Pflichtverstoß.

b) Sonderbehandlung der Schlechterfüllung während des Gesetzgebungsverfabrens

Fraglich ist, ob neben diesen Schutz durch das Fristsetzungserfordernis beim Schadensersatz statt der Leistung weiterer Schuldnerschutz treten sollte. So wurde es für den Hauptfall - die Schlechterfüllung - in der Gesetzgebungsgeschichte in der Tat vorgeschlagen. Die Schuldrechtskommission wollte noch Schadensersatz statt der Leistung auch bei Schlechterfüllung parallel zu den Rücktrittsvoraussetzungen regeln und zwar im genannten Sinne: Dem Schuldner sollte die zweite Chance durch Fristsetzung verbürgt werden, mehr Schutz hingegen nicht. Die Leistungsstörungskommission (2001) schlug demgegenüber vor, die Selbstständigkeit der Schlechterfüllung (wieder) herzustellen. Schadensersatz statt der Leistung sollte bei Schlechterfüllung, anders als bei Nichterfüllung oder Unmöglichkeit, nur geschuldet sein, wenn es dem Gläubiger unzumutbar wäre, sich allein mit dem Aufstockungsschadensersatzanspruch („neben der Leistung“, $\$ 280$ Abs. 1 BGB) zu begnügen. Diese Lösung hätte also für den Hauptfall gegolten, die Schlechterfüllung, nicht nur für die engen Ausnahmefälle einer Teilleistung und eines Pflichtverstoßes gegen nicht leistungsbezogene Nebenpflichten, für die diese Unzumutbarkeitsausnahme auch in der verabschiedeten Gesetzesfassung beibehalten wurde (vgl. unten c). Mit dem Vorschlag der Leistungsstörungskommission (2001) wäre nicht nur die Einheitlichkeit in der Behandlung aller wichtigen Verstoßtatbestände beim Schadensersatz statt der Leistung aufgelöst worden, sondern auch der Parallellauf zum Rücktrittsrecht. Denn die Kommission schlug das Unzumutbarkeitserfordernis zwar für den Schadensersatz statt der Leistung, nicht jedoch für den Rücktritt vor, und dies für die Schlechterfüllung, jedoch nicht die großen anderen Verstoßtatbestände: Verspätung und Nichterfüllung.

Dies beruht auf einer dogmatisch zwar vertretbaren, die praktische Bedeutung jedoch ausblendenden Kategoriebildung. Canaris sieht die Reihenfolge der Rechtsbehelfe nach ihrer (jeweils zunehmenden) Eingriffsintensität für den Schuldner so: Auf den Rücktritt folgen, bereits eingriffsintensiver, der Schadensersatz neben der Leistung und zuletzt der Schadensersatz statt der Leistung. ${ }^{51}$ So gesehen, mag es plausibel sein, beim Schadensersatz statt der Leistung viel mehr - zusätzlich Unzumutbarkeit - zu fordern als beim Rücktritt. Dogmatisch liegt solch eine Reihenfolge nahe (wenn man das Verschul-

${ }^{51}$ Canaris, JZ 2001, 499, 514. 
denserfordernis befürwortet), denn nur bei den letzten zwei Ansprüchen wird Vertretenmüssen gefordert. Wirtschaftlich ist die Reihenfolge jedoch auf Grund der genannten Entwertung und des Wegfalls des Geschäftsgewinns typischerweise eine andere: Der Schadensersatz neben der Leistung wirkt häufig weniger belastend, Rücktritt und Schadensersatz statt der Leistung schwerer, beide, abgesehen von Gewinnausfall und Folgeschäden, regelmäßig gleich intensiv.

Eine der wichtigsten Korrekturen in den letzten Gesetzgebungswochen ging dahin, die Sonderbehandlung der Schlechterfüllung beim Schadensersatz statt der Leistung aufzugeben (und damit zum Modell der Schuldrechtskommission zurückzukehren).

c) Sonderbehandlung in der verabschiedeten Fassung zurückgedrängt $\left(\iint 281 f . B G B\right)$

Im Schuldrechtsmodernisierungsgesetz wird die Schlechterfüllung auch für den Schadensersatz statt der Leistung dem allgemeinen Regime unterworfen, so dass auch insoweit nur Fristsetzung gefordert wird, nicht ein besonderes Gläubigerinteresse. ${ }^{52}$ Fristsetzungsfragen, insbesondere der Einfluss der Kaufrechts-Richtlinie, ${ }^{53}$ müssen hier nicht diskutiert werden. Für die Kategorienbildung sind sie nicht relevant. ${ }^{54}$

Wichtig für das System ist demgegenüber die Frage, wie weit die Sonderregel nach $₫ 282 \mathrm{BGB}$ reicht. Offenbar ist eine Verschärfung gegenüber $\ 281$

52 Huber/Faust (Fn. 32), 127; Lorenz/Riehm (Fn. 12) 112; MünchKommBGB/ Ernst $\$ 281 \mathrm{Rn} .123,136$; nicht durchgesetzt hat sich in der Gesetzgebungsgeschichte der Ansatz von Canaris, JZ 2001, 499, 513, der tendenziell die Schlechterfüllung auch konzeptionell als Teilerfüllung ansah (noch zu $\$ 281$ Abs. 1 S. 3 der konsolidierten Fassung des Diskussionsentwurfes); ebenso Canaris selbst: Die Neuregelung des Leistungsstörungs- und des Kaufrechts - Grundstrukturen und Problemschwerpunkte, in: E. Lorenz (Hrsg.), Karlsruher Forum 2002: Schuldrechtsmodernisierung 2002, 5, $33 \mathrm{f}$.

53 Die Richtlinie fordert nur den Ablauf einer angemessenen Frist, nicht deren Setzung durch den Gläubiger (vgl. dort Art. 3 Abs. 3 S. 3 und Abs. 5). Das deutsche Recht kann für den Rücktritt des Verbrauchers im Kauf- und Werkvertragsrecht wohl richtlinienkonform ausgelegt werden, weil der deutsche Gesetzgeber ersichtlich nicht gegen die Kaufrechts-Richtlinie verstoßen wollte. Dass der deutsche Gesetzgeber dennoch nicht korrekt umsetzte (Umsetzung muss rechtssicher sein), steht auf einem anderen Blatt. Zur Frage, ob die Richtlinienstandards für den Schadensersatzanspruch überhaupt relevant sind, vgl. nur Fn. 8.

${ }^{54}$ Eine unterschiedliche Ausgestaltung je nachdem, ob der Gläubiger ein Verbraucher oder ein beruflich Tätiger ist, erscheint beim Fristsetzungserfordernis auch (ausnahmsweise) erwägenswert. Angedacht etwa bei Canaris, JZ 2001, 499, 510 f.; vgl. auch Kropholler (Fn. 34), $\$ 281$ Rn. 8 (jedenfalls zu knappe Fristsetzung in angemessene umzudeuten); allgemein vgl. Nachw. oben Fn. 6. 
BGB intendiert, ${ }^{55}$ weitgehend parallel zu der in $₫ 281$ Abs. 1 S. 2 BGB enthaltenen. Theoretisch gilt also wiederum das Fristsetzungserfordernis $(\mathbb{} 281$ Abs. 1 S. 1 BGB), daneben statt des bloßen Unerheblichkeitsvorbehalts ( $\$ 281$ Abs. 1 S. 3 BGB), weitergehend, ein Unzumutbarkeitsvorbehalt. Nicht gänzlich geklärt ist der Umfang des Verweises: Einerseits wird $\$ 241$ Abs. 2 BGB, auf den $\$ 282$ BGB verweist, häufig dahin verstanden, dass hier die leistungsbezogenen und die nicht leistungsbezogenen Nebenpflichten gleichermaßen angesprochen seien, ${ }^{56}$ also einerseits etwa Pflichtverstöße beim Verpacken, bei der Anleitung zum Umgang mit der empfangenen Leistung, bei der Aufklärung etc., andererseits Pflichtverstöße bei Rücksichtnahme auf das sonstige Vermögen und sonstige Interessen des Gläubigers, die unabhängig vom Vertrag vorhanden waren (Umstoßen der Vase, Ausplaudern von Geschäftsgeheimnissen, die bei der Verhandlung in Erfahrung gebracht wurden). Umgekehrt wird für $\$ 282$ BGB überwiegend davon ausgegangen, dass die hier verschärften Anforderungen nur bei Verstoß gegen nicht leistungsbezogene Nebenpflichten eingreifen. ${ }^{57}$ Mit anderen Worten: Der Verweis auf $\$ 241$ Abs. 2 BGB wird nur auf den einen Zweig der dort (nach Mehrheitsansicht) angesprochenen (Neben-) Pflichten bezogen, nämlich auf die nicht leistungsbezogenen Nebenpflichten.

Im Ergebnis ist dem zuzustimmen und entspricht dies auch dem internationalen Modell (beim Rücktritt). Auf dem Hintergrund der Strukturen des Bürgerlichen Rechts erscheint folgende Überlegung erheblich: Unzumutbarkeit spielt ein zweites Mal eine Rolle im Leistungsstörungsrecht, nämlich im Rahmen von $\$ 275$ Abs. 3 BGB, der sich gerade durch dieses Kriterium von der Regelung in $\$ 275$ Abs. 2 BGB abhebt. Medicus erklärt das folgendermaßen: „Die Sängerin braucht nicht wie versprochen aufzutreten, wenn sie bei ihrem ... im Sterben liegenden Kind bleiben will. Hier kann man das Verlassen des Kindes ... schwerlich als ,Aufwand“ ... bezeichnen. Und schon gar nicht kann man [es] ... zu dem (regelmäßig materiellen) Interesse des Veranstalters in Beziehung setzen. Daher bleibt nur der Rückgriff auf die (relativ unbestimmte) Zumutbarkeit. “58 Auch nicht leistungsbezogene Schutzpflichten betreffen Positionen, die in das Äquivalenzverhältnis zwischen den Vertragsleistungen nicht einbezogen waren. Dieser Gesichtspunkt ist erheb-

55 Vgl. BT-Drucks. 14/6040, 141 f.; MünchKommBGB/Ernst, $\$ 282$ Rn. 1, 5.

${ }^{56}$ Kropholler (Fn. 34), $\$ 241$ Rn. 2 (Beispiele); MünchKommBGB/Roth $\$ 241$ Rn. 31 ff., bes. 67 ff.; Huber/Faust (Fn. 32), 68; Lorenz/Riehm (Fn. 12) 177-182; demgegenüber werden nur die nicht leistungsbezogenen Nebenpflichten unter $\$ 241$ Abs. 2 BGB gefasst von Palandt/Heinrichs, $\$ 241$ Rn. 6.

57 BT-Drucks. 14/6040, 141; BT-Drucks. 14/7052, 281; Kropholler (Fn. 34), $\$ 282$ Rn. 1; MünchKommBGB/Ernst $\$ 282$ Rn. 1; Huber/Faust (Fn. 34), 131.

${ }^{58}$ Medicus (Fn. 16), Rn. 375. 
lich für die Abgrenzung. So mag ein Gutachter ein vollständig zufriedenstellendes Gutachten erstellt haben, im Gespräch dann jedoch den Auftraggeber beleidigt und die mit dem Gutachten verfolgten $Z$ wecke angezweifelt haben. Man mag daran denken, $\$ 282$ BGB heranzuziehen und Unzumutbarkeit zu prüfen. Maßgeblich für die Abgrenzung muss jedoch sein, ob der Gutachter das Gutachten bei Verhandlungen erläutern sollte oder zumindest diese Möglichkeit bestand. Ist Unzumutbarkeit ein Notnagel, heranzuziehen bei fehlender Vergleichbarkeit der gegenläufigen Interessen und im Gesetzgebungsverfahren zu Recht abgeschafft als Kriterium für die Beurteilung der Schlechtleistung, so muss dies in die Wertung einfließen. Wenn die Pflichtverletzung nur irgend wie auch leistungsbezogen sein kann - etwa vorliegend befürchten lässt, dass der Gutachter in einer Verhandlung die Negativbemerkungen wiederholt -, ist (neben Fristsetzung oder Abmahnung) allein die Unerheblichkeitsausnahme des $\$ 281$ BGB heranzuziehen. Grundsätzlich genügt die zweite Chance, mehr sollte der gegen seine Pflichten verstoßende Schuldner nicht erhalten, dann muss das Wahlrecht und damit auch das Druckpotenzial des Gläubigers greifen. Freilich wird bei Unzumutbarkeit regelmäßig $\ 281$ Abs. 2, 2. Alt. BGB eingreifen, die Fristsetzung also (ausnahmsweise) überflüssig sein. Der Unterschied zwischen den Voraussetzungen nach $\$ 281$ BGB (für alle irgendwie leistungsbezogenen Pflichten) und denen nach $\$ 282$ BGB (für alle ausschließlich nicht leistungsbezogenen Nebenpflichten) liegt also in der praktischen Anwendung darin, dass in $\$ 281$ BGB Fristsetzung und -ablauf oder Unzumutbarkeit ( $\$ 281 \mathrm{Abs}$. 2, 2. Alt. BGB) gefordert wird, in $\$ 282$ BGB erstere hingegen nicht genügt.

\section{Vertraglicher Schadensersatz und Verzug}

Wenn schon weder die Unmöglichkeit (oben 2. und 3.) noch die Schlechterfüllung (oben 4.) eigenen Regeln folgen, so unterliegt doch wenigstens die verzögerte Erfüllung offensichtlich schadensersatzrechtlich gesonderten, abweichenden Vorschriften. Dies wurde der Schuldrechtskommission vorgehalten, Differenzierungen hätte sie durch die Hintertür doch wieder eingeführt. ${ }^{59}$

\section{a) Ein zweiter Schadensersatzanspruch mit Zusatzanforderungen}

In der Tat entstammen die zwei Eckpfeiler der Regelung dem Entwurf der Schuldrechtskommission: sowohl der Verweis in $\$ 280$ Abs. 2 und 3 BGB auf zwei Schadensersatzgruppen, die Zusatzanforderungen unterworfen werden,

59 Vgl. etwa Schapp, Empfiehlt sich die „Pflichtverletzung“ als Generaltatbestand des Leistungsstörungsrechts?, JZ 2001, 583, 586. 
als auch die Ausgestaltung von $\$ 286$ Abs. 1 und 2 BGB. ${ }^{60}$ Zunächst zum Ersten: Die doppelte Verweisung in $\$ 280$ Abs. 2 und 3 BGB erweckt den Anschein, als würden zwei Schadensersatzgruppen weitgehend parallel behandelt: der Schadensersatz statt der Leistung (\$\$281-284 BGB) und der Schadensersatz aus Verzug ( $\$ 286 \mathrm{ff}$. BGB). Die Parallelität erscheint dadurch noch verstärkt, dass das Hauptzusatzerfordernis jeweils darin besteht, dass der Gläubiger „nachhaken“ muss: durch Fristsetzung bzw. durch Mahnung. Als erster großer Unterschied springt freilich spätestens bei zweitem Hinsehen ins Auge, dass beim Verzugsschaden auch und gerade der Schadensersatz neben der Leistung, der Aufstockungsschaden ( $\$ 280$ Abs. 1 BGB), diesen $\mathrm{Zu}$ satzvoraussetzungen unterworfen wird. Wenn also bisher mühsam hergeleitet werden musste, dass in Wahrheit beim Schadensersatz statt der Leistung alle klassischen Verstoßtatbestände - Nichterfüllung, Unmöglichkeit, Schlechterfüllung - gerade nicht, wie die Aufteilung in $\$ \$ \$ 281-283$ BGB suggeriert, unterschiedlichen Voraussetzungen folgen und dass nur in den Randbereichen (Teilleistung und Verstoß gegen nicht leistungsbezogene Pflichten) Abweichungen zu konstatieren sind, so zeigt sich hier nun das Umgekehrte: Gerade beim Schadensersatz neben der Leistung, der in $\$ 280$ Abs. 1 BGB so kompakt, so homogen für alle Pflichtverstöße geregelt erscheint, wird nun zwischen den klassischen Verstoßtatbeständen unterschieden: zwischen (Teil-)Nichterfüllung, Schlechterfüllung, auch Verstoß gegen leistungsbezogene oder nicht leistungsbezogene Nebenpflichten auf der einen Seite und Verzug auf der anderen.

Die beiden Schadensersatzgruppen, für die $\$ 280$ in Abs. 2 und 3 BGB Zusatzerfordernisse aufstellt, sind also denkbar heterogen. In Abs. 2 wird Abs. 1 modifiziert, indem für den dort geregelten Aufstockungsschadensersatz in einer Fallgruppe die Mahnung als Zusatzvoraussetzung angekündigt wird. In Abs. 3 wird für eine andere Rechtsfolge - den Schadensersatz statt der Leistung, also den Substitutionsschadensersatz - die Fristsetzung als Zusatzvoraussetzung angekündigt, weil diese Rechtsfolge ungleich einschneidender ist („zweite Chance“).

$60 \$ 286$ Abs. 3 BGB enthält natürlich die Umsetzung der Zahlungsverzugs-Richtlinie (Richtlinie 2000/35/EG des Europäischen Parlaments und des Rates zur Bekämpfung von Zahlungsverzug im Geschäftsverkehr vom 29.6.2000, ABl.EG 2000 L 200/35) und ist vorliegend, zumal nach der Korrektur der „gesetzgeberischen Fehlleistung“ in \284 Abs. 3 BGB a.F., nicht wichtig. 
b) System weitgehend obne Zusatzanforderungen denkbar: Fälligkeitszins bei Beachtlichkeit weitergehender Verzugsschäden

Wenn der Schuldrechtskommission also zumindest für den Auffüllungsschadensersatz vorgeworfen werden kann, dass sie hier eine Differenzierung nach Verstoßformen doch wieder durch die Hintertür einführt, so werden dafür - und damit für die Aufgliederung nach Verstoßtatbeständen - fundamentale Billigkeitsüberlegungen als maßgeblich angesehen. ${ }^{61}$ Sachlogisch zwingend ist die - in der Tat schon im Entwurf der Schuldrechtskommission zu findende - Ungleichbehandlung verschiedener Verstoßtatbestände jedoch keineswegs. Ganz im Gegenteil ist die Gleichbehandlung des Verzugsschadens zumindest für den Zahlungsanspruch im Inland vehement gefordert worden und rechtsvergleichend üblich - nämlich als marktbezogener Fälligkeitszins. Kindler hat die rechtspolitische Überlegenheit eines solchen m.E. überzeugend belegt, und zugleich auch, dass er rechtsvergleichend in Europa weitestgehend zu finden ist, wie bei uns im zweiseitigen Handelsgeschäft, nur im Ausland häufig eben auch mit marktüblichen Sätzen. ${ }^{62}$ Solch ein Fälligkeitszins würde dann den normalerweise zu erwartenden Verzugsschaden darstellen. Und für einen zusätzlichen, speziell bei diesem Gläubiger zu erwartenden Schaden ließe sich das Erfordernis einer Mahnung auch im veränderten System gut rechtfertigen: als Hinweis auf eine gesteigerte persönliche Schadensneigung, auf die hinzuweisen der Gläubiger verpflichtet werden müsste, weil er insoweit besseren Zugang zur relevanten Information hat, was dogmatisch auch unter Hinweis auf $₫ 254$ BGB systemstimmig zu begründen wäre. ${ }^{63}$ Marktzinsorientierter Fälligkeitszins und eine aus $\ 254$ BGB herzu-

${ }^{61}$ Huber, Leistungsstörungen, Bd. I, 1999, 412-414 ( $\$ 17$ I 1); auch Medicus (Fn. 16), Rn. 399; in anderem Zusammenhang verwendet Canaris auch prägnant den Begriff der sachlogischen Struktur (JZ 2001, 499, 502).

${ }_{62}$ Kindler, Gesetzliche Zinsansprüche im Zivil- und Handelsrecht - Plädoyer für einen kreditmarktorientierten Fälligkeitszins, 1996, 150-182, bes. 165-169, 303 f. et passim; grundsätzlich vergleichbar in der Kritik: für die Höhe: Medicus, Vorschläge zur Überarbeitung des Schuldrechts: Das allgemeine Recht der Leistungsstörungen, NJW 1992, 2384, 2386; Roll, Die Höhe der Verzugszinsen, DRiZ 1973, 339, 342; Bundesminister der Justiz (Fn. 12), 115-117; für den Fälligkeitszins: Peters, Der Zinssatz des $\$ 288$ I 1 BGB, ZRP 1980, 90, 93. Die Einführung eines marktbezogenen Zinses (insbesondere in $\$ 288$ Abs. 1 BGB iVm $\$ 247$ BGB) wird diesem Anliegen teilweise gerecht, dieser Zinssatz gilt jedoch nicht für den (handelsrechtlichen) Fälligkeitszins und im Bürgerlichen Recht fehlt noch immer der Fälligkeitszins (vgl. aber im Folgenden zu Gestaltungsmöglichkeiten).

$63 \$ 254$ Abs. 2 S. 1 BGB statuiert bekanntlich die Obliegenheit, den Schädiger auf die Gefahr eines besonders hohen Schadens oder einer für ihn nicht erkennbaren Schadenspositon aufmerksam zu machen, vgl. etwa MünchKommBGB/Oetker ${ }_{2} \mathbb{} 254$ Rn. 70-74. 
leitende Hinweispflicht bei weiterreichender Schadensneigung (Hinweis durch Mahnung) - sachlogisch wäre solch eine Lösung durchaus ebenfalls, mehr noch: Sie wird von vielen vehement gefordert.

\section{c) Auch realisiertes System mit nur schwachen Zusatzanforderungen}

Das realisierte System kennt den Fälligkeitszins jedoch nicht durchgehend und dieser ist auch nicht marktzinsbezogen ausgestaltet. Der Auffüllungschadensersatz („neben der Leistung“, $\$ 280$ Abs. 1 BGB) unterliegt also bei Verzug - zwar nicht sachlogisch zwingend, jedoch eben de lege lata - anderen Voraussetzungen als bei allen anderen Vertragspflichtverletzungen. Gefragt werden muss freilich, wie stark diese Zusatzvoraussetzungen tatsächlich wiegen.

Meist werden die Zusatzerfordernisse beim Verzug (Auffüllungsschaden) und diejenigen im Regime der $\$ \$ \$ 281-283$ BGB (Substitutionsschaden) parallel gesehen. ${ }^{64}$ Das scheint mir die Sonderstellung weit überzubetonen. Formal steht der Auffüllungsschadensersatz aus Verzug im Tatbestand zwar dem Schadensersatz statt der Leistung näher, praktisch jedoch, bei guter Vertragsgestaltung, ungleich näher allen anderen Fällen eines Auffüllungsschadensersatzes. Bei Vertragsgestaltung lege artis geht er in ihnen sogar vollständig auf.

Dies liegt nicht so sehr daran, dass eine Mahnung nach $\$ 286$ BGB weniger Zuwarten verlangt als Fristsetzung nach $\$ 281$ BGB. Die Rechtsfolge tritt mit Mahnung, also mit Erklärungszugang, nicht erst mit Fristablauf ein. ${ }^{65} \mathrm{Un}$ gleich wichtiger sind jedoch die Unterschiede bei den Ausnahmen: Die gleichen Ausnahmen gelten beim Mahnerfordernis hier und Fristsetzungserfordernis dort, bei ernsthafter und endgültiger Erfüllungsverweigerung und sonst bei überwiegendem Gläubigerinteresse ( $\$ 281$ Abs. 2 BGB und gleichlautend $\$ 286$ Abs. 2 Nr. 3 und 4 BGB). Bei der Mahnung kommen jedoch zwei Ausnahmen nach $\$ 286$ Abs. 2 Nr. 1 und 2 BGB hinzu: die kalendermäßige Bestimmtheit, die schon nach $\$ 285$ BGB a.F. das Mahnerfordernis entfallen ließ, und die kalendermäßige Berechenbarkeit nach einem bestimmten Ereignis. Letztere ist neu und geht auf den Vorschlag der Schuldrechtskommission zurück. Diese neue Ausnahme führt nun jedoch zugleich dazu, dass (ohne Aufbauen geschäftsschädigender Drohgebärden) jeder Vertragspartner durch Vertragsgestaltung lege artis, etwa durch Festlegungen wie

${ }^{64}$ Kropholler (Fn. 34), $\$ 280$ Rn. 1, 7-9; MünchKommBGB/Ernst $\$ 280$ Rn. 1, 3.

${ }^{65}$ Nach zutreffender und auch ganz überwiegender Meinung umfasst denn Fristsetzung auch Mahnung: Kropholler (Fn. 34), \$281 Rn. 9; Larenz, Lehrbuch des Schuldrechts, Band I, Allgemeiner Teil ${ }^{14}, 1987$, $\$ 23$ I a; MünchKommBGB/Ernst $\$ 286$ Rn. 48; Palandt/Heinrichs, $\$ 286$ Rn. 17; zumindest im Regelfall nach Canaris, JZ 2001, 499, 515. 
Zahlung „14 Tage nach Lieferung“ oder „zwei Wochen nach Leistungsabruf“ etc., ${ }^{66}$ einen Verzugseintritt ohne Mahnung sicherstellen kann. Zusatzerfordernissen unterworfen ist der Auffüllungsschaden aus Verzug - parallel zum Schadensersatz statt der Leistung nach $\$ 281$ BGB und abweichend vom allgemeinen Regime des Auffüllungsschadensersatzanspruches nach $₫ 280$ Abs. 1 BGB - überwiegend nur für den „schludrigen“ Vertragsgestalter. Die sonstigen Zusatzerfordernisse für den Schadensersatz statt der Leistung ( $\mathbb{S} 281$ Abs. 1 S. 2 und 3, 282 BGB) gelten ohnehin nicht.

\section{Fazit: Rechtsfolgenorientierung statt Tatbestandsorientierung}

Interpretiert man das Gesagte, fällt zweierlei auf: Während das alte Recht primär nach Tatbeständen - Unmöglichkeit, Schlechterfüllung, Verzug - unterschied, unterscheidet das neue nach Rechtsfolgen. Letztlich wird damit nachvollzogen, was insbesondere Medicus mit seiner Ordnung nach Anspruchsgrundlagen in der Darstellung des Bürgerlichen Rechts vorgedacht hatte. ${ }^{67}$ Vor allem jedoch: Begehren (Rechtsfolgen) sind die Kategorie, auf die der „Kampf ums Recht“ abzielt, wie der Vater der Interessenjurisprudenz titelte, ${ }^{68}$ und worum es bei der Interessen- und Wertungsjurisprudenz allgemein und beim typischen Anspruchssteller in der Tat primär geht. Dieser veränderte Ansatz ist auch in einem weiteren Aspekt Ausdruck eines veränderten Verständnisses. 1900, in einer Welt, in der Handlungen und Verantwortlichkeiten noch individualisierbar erschienen, war die einzelne, individualisierte Fehlleistung Ausgangspunkt. Von der Freiheitsethik her musste dies möglicherweise sogar zwingend erscheinen. Hundert Jahre später ist das Wirklichkeit, was Ortega y Gasset als den Aufstand der Massen beschrieb. ${ }^{69}$ Recht muss den Massenverkehr adäquat erfassen. Im Deliktsrecht werden zunehmend Versicherungslösungen gefordert und dringen wohl auch in immer mehr Teilbereichen vor. Im Vertragsrecht ist es folgerichtig, nicht mehr an die einzelne Handlung, die Verstoßkategorie, anzuknüpfen und sie einzuordnen, sondern die zeitlich und qualitativ nicht vollständig planmäßige Durchführung - unabhängig noch vom Vertretenmüssen oder gar Verschulden - als

${ }^{66}$ Dazu, dass solche Formulierungen unter $\$ 286$ Abs. 2 Nr. 2 BGB zu fassen sind: Huber/Faust (Fn. 32), 87; MünchKommBGB/Ernst \$286 Rn. 87; Lorenz/Riehm (Fn. 12) 133; Palandt/Heinrichs, $\$ 286$ Rn. 17.

67 Medicus, Bürgerliches Recht, 1. Aufl. 1968 (heute 19. Aufl. 2002). Das Lehrbuch von Medicus zum Schuldrecht (Allgemeiner Teil) wird diesen Schritt vielleicht in den nächsten Auflagen nachvollziehen.

${ }^{68}$ Ihering, Kampf ums Recht, 1872.

69 Ortega y Gasset, La rebelión de las masas, 1929 (deutsch: Der Aufstand der Massen, erstmals 1931). 
zentrales, ja einziges Aufgreifkriterium zugrunde zu legen. Dies genügt der anderen Vertragsseite für den Rücktritt und - angesichts $\$ 280$ Abs. 1 S. 2 BGB - regelmäßig auch für den Schadensersatz. Beim Ausgehen von der Rechtsfolge wird also auf das eigentliche Anliegen der Rechtsunterworfenen unmittelbarer eingegangen, und das weitestgehende Fallenlassen von Unterscheidungen nach verschiedenen Verstoßformen entspricht den Anforderungen der Rechtssicherheit und Vorhersehbarkeit in einer zunehmend durch anonyme und Massentransaktionen geprägten Welt. Bei den Rechtsfolgen ist dann in der Tat entscheidend, ob der Leistende seine Leistung zurücknehmen muss, weil er diese damit selbst häufig entwertet erhält und meist parallel auch seinen Geschäftsgewinn verliert. In dieser Situation ist es richtig, dem Schuldner durch Fristsetzung eine zweite Chance zu geben, umgekehrt jedoch dem Gläubiger ein Druckpotential zu erhalten, wenn der Schuldner dennoch nicht vertragsadäquat leistet.

\section{Stellung im sonstigen Leistungsstörungsrecht}

Das Thema Schadensersatz aus Vertrag hat Bezugspunkte vor allem zu zwei Instituten. Sie wurden bereits als die anderen beiden Eckpfeiler des Leistungsstörungsrechts benannt: der Entfall der Primärleistungspflicht und die Rückabwicklung durch Rücktritt als die zweite Form eines Sekundäranspruchs. Für diese Institute können vorliegend nur Ergebnisse benannt, nicht entwickelt werden. Teils wurden sie bereits angedeutet.

\section{a) Wegfall der Primärleistungspflicht}

Auch der Wegfall der Primärleistungspflicht ist eine Kategorie, für die sich ein Einheitskonzept, anders als im alten Recht, durchgesetzt hat: Unstreitig unerheblich ist heute, ob die Unmöglichkeit subjektiv oder objektiv ist, anfänglich oder nachträglich, verschuldet oder nicht. Nur ein letzter Schritt ist noch zu gehen: Beim Wegfall der Primärleistungspflicht handelt es sich auch um eine Kategorie, die nicht von der Verstoßart abhängt oder abhängen muss sondern vom Gedanken des nemo obligetur und - im gegenseitigen Vertrag vom Äquivalenzprinzip. ${ }^{70}$ In der Tat erscheint es angezeigt, $\$ 275$ BGB durchaus auch auf Schlechterfüllung, die absehbar nicht kuriert werden kann, und danach etwa auf die Nacherfüllung anzuwenden. ${ }^{71}$ Und für die Zulas-

$70 \mathrm{Zu}$ diesen Aspekten als den tragenden für $\$ 275 \mathrm{BGB}$ bzw. $\$ \$ 323 \mathrm{ff}$. BGB (verschuldensunabhängig): MünchKommBGB/Ernst $\$ 275$ Rn. 30; weiterhin erhellend Bundesminister der Justiz (Fn. 12), 117-121; bzw. Bydlinski, System und Prinzipien des Privatrechts, 1996, 182; Canaris, JZ 2001, 499, 522.

${ }^{71}$ Ausdrücklich so Kommentar C zur Vorschrift in Art. 9:102 PECL (Fn. 50), auf 
sung des Rücktritts unabhängig vom Verschulden ( $\$ \$ 323 \mathrm{f}$. BGB) ebenso wie für den Entfall der Gegenleistungspflicht ipso iure nach $\$ 326$ BGB spricht gleichermaßen das Äquivalenzprinzip. Dass dann der Wegfall der Primärleistungspflicht in dem einen Fall eine Gestaltungserklärung des Gläubigers voraussetzt, in dem anderen ipso iure eintritt, betrifft eine eher nebensächliche Ausübungsmodalität und ist noch nicht einmal denknotwendig so..$^{72} \mathrm{Nur}$ in dieser Ausübungsfrage divergiert also die rechtliche Behandlung in Fragen des Wegfalls der Primärleistungspflicht beim Verstoßtatbestand Unmöglichkeit von derjenigen bei den sonstigen Verstoßtatbeständen, vor allem Verzug, Schlechterfüllung und Verstoß gegen Nebenpflichten. Ansonsten könnte die Rechtsfolge des $\$ 326$ BGB (Auflösungsrecht auch ohne Fristsetzung) wiederum unschwer auf $\$ 323$ Abs. 2 BGB gestützt werden. Das Verhältnis ist vergleichbar dem zwischen $\ 283$ BGB und $\$ 281$ BGB (oben 2.).

\section{b) Rücktrittsrecht}

Sieht man von diesen Ausübungsmodalitäten ab, ist der Rücktritt bzw. Entfall der Primärleistung(en) und der Schadensersatz statt der Leistung in $\mathbb{\int} 281-283$ BGB und $\$ \$ 323 \mathrm{f}$. BGB (bis hin zu $\ 326 \mathrm{BGB}$ ) streng parallel geregelt. ${ }^{73}$ Das ist überzeugend, weil der Schuldner bei Wegfall seiner Primärleistung, wie oben beschrieben, zweifach belastet ist und gerade in diesem maßgeblichen Punkt Rücktritt und Schadensersatz statt der Leistung parallel laufen. Den vollständigen Parallellauf wollte die Schuldrechtskommission, die Leistungsstörungskommission (2001) hat im Kernstück, der Schlechterfüllung, hingegen Rücktritt und Schadensersatz statt der Leistung auseinander entwickelt, ${ }^{74}$ das Gesetz hat wieder den völligen Gleichlauf hergestellt

die sich die Leistungsstörungskommission (2001) und der Gesetzgeber berief; etwa Canaris, JZ 2001, 499, 505. Nichterfüllung im internationalen Kontext ist eben gleichzusetzen mit „Pflichtverletzung“ im deutschen Sprachgebrauch und gerade nicht mit „Nichterfüllung" im deutschen Sprachgebrauch, was bei Anlegung der deutschen Kategorien an internationale Texte übersehen werden kann. Dezidiert für ein enges Verständnis der Unmöglichkeit und für die Maßgeblichkeit dieser engen Kategorie in Fragen des Wegfalls der Primärleistungspflicht: Canaris, Zur Bedeutung der Kategorie "Unmöglichkeit“ für das Recht der Leistungsstörungen, in: Schulze/Schulte-Nölke (Fn. 6), 43.

72 In der Tat sah die Schuldrechtskommission noch für alle Fälle den Rücktritt vor: Bundesminister der Justiz (Fn. 12), $167 \mathrm{f}$. ( $\$ 323$ des Entwurfs).

73 Auch beim Rücktritt hat sich - im Vergleich mit dem alten Recht - ein Einheitskonzept durchgesetzt: Irrelevant ist heute insbesondere die Unterscheidung, ob die Pflichtverletzung verschuldet ist oder nicht und ob es sich um einen Verstoß gegen eine Hauptpflicht handelt oder nicht (beides war von Relevanz nach altem Recht).

${ }_{74}$ Vgl. $\$ 283$ Abs. 1 S. 2 und Abs. 3 S. 1 des Entwurfs der Schuldrechtskommission, die eine zusätzliche Zumutbarkeitsvoraussetzung nur für die Teilleistung und Rückge- 
( $\int \$ 323$ f. BGB und $\int \$ 281$ f. BGB). Die drei Ausnahmefälle, in denen die Tatbestandsmerkmale nicht auch formal parallel laufen, erweisen sich materiell nicht als solche: Die zusätzliche Ausnahme vom Fristsetzungserfordernis ( $\$ 323$ Abs. 2 Nr. 2 BGB) bezeichnet in Wirklichkeit eine vertraglich vereinbarte Ausnahme vom Fristsetzungserfordernis und diese ist selbstverständlich auch beim Schadensersatz statt der Leistung möglich. $\$ 323$ Abs. 4 BGB, nach dem schon vor Fälligkeit Rücktritt gewählt werden kann, wenn sicher ist, dass die Rücktrittsvoraussetzungen eintreten werden, ist wichtig für die vom Rücktrittsberechtigten zu erbringende Leistung. Er soll diese gar nicht erst erbringen müssen. Beim Schadensersatz statt der Leistung bleibt die Verpflichtung zu dieser Leistung demgegenüber dauerhaft bestehen (nur die Primärleistungspflicht des vertragsbrüchigen Schuldners entfällt). Und die Leistung der anderen Seite - oder Ersatz hierfür - kann der Gläubiger selbstverständlich erst bei Fälligkeit fordern. Zuletzt wird auch $₫ 323$ Abs. 6 BGB zu Recht in $\$ 281$ BGB hineingelesen ${ }^{75}$ - jedenfalls über $\$ 254$ BGB.

Mit der Parallelität zwischen Schadensersatz statt der Leistung und Rücktritt ist zugleich ein weiterer Punkt zum Verhältnis dieser beiden Leistungsstörungsrechte zueinander angesprochen: Letztlich geht es bei Schadensersatz neben der Leistung, Schadensersatz statt der Leistung (unter Wegfall der Primärleistung des Verpflichteten) und (Aufstockungs-) Schadensersatz neben dem Rücktritt ( $\ 325$ BGB, unter Wegfall der Primärleistungen beider Seiten) um drei verschiedene Möglichkeiten des Verpflichteten, sich „seinen“ Schadensersatzanspruch zusammenzustellen: indem er es bei beiden Primärleistungen belässt und nur den Aufstockungsbetrag fordert, indem er es bei der eigenen Primärleistung belässt, jedoch den vollen Ersatz für die Primärleistung der anderen Seite fordert, oder indem er auch die eigene Primärleistung nicht erbringen will (weil ihm etwa das Tauschgut, das er hingeben muss, lieb ist und er es nur gegen ein anderes begehrtes Stück hingeben wollte), dafür jedoch auch nur einen geringeren (Aufstockungs-)Schadensersatzanspruch geltend machen kann. Unter diesem Gesichtspunkt erschiene es naheliegend, den Gehalt von $\$ 325$ BGB im Zusammenhang mit dem Schadensersatz zu regeln, und nicht beim Rücktritt. Letztlich rührt das Zuordnungsdilemma freilich von einer anderen Eigenart des deutschen Leistungsstörungsrechts her: Hier werden Rechtsfolgen, die auch im einseitig verpflichtenden Vertrag

währansprüche vorsahen (Bundesminister der Justiz [Fn. 12], 290) und \$281 Abs. 1 S. 3 des Entwurfs der Leistungsstörungskommission (2001), der eine zusätzliche Zumutbarkeitsvoraussetzung für die Teilleistung und die Schlechtleistung vorsah (JZ 2001, 525). Für den Rücktritt sahen hingegen beide bei der Schlechterfüllung nur das Fristsetzungserfordernis vor (jeweils $\$ 323$ Abs. 1, a.a.O. S. 292 bzw. 527).

75 BT-Drucks. 14/6040, 187; Kropholler (Fn. 34), $\$ 323$ Rn. 4; Palandt/Heinrichs, $\$ 323$ Rn. 18. 
(oder Sonderrechtsverhältnis) Bedeutung haben (Schadensersatz), von Rechtsfolgen getrennt, die allein im zweiseitig verpflichtenden Vertrag zum Tragen kommen (Rücktritt), obwohl beide im Normalfall, nämlich dem zweiseitig verpflichtenden Vertrag, für den Berechtigten die Alternativen sind, zwischen denen er zu wählen hat.

\section{Nur eine kategoriale Änderung bei Inhalt und Höbe des vertraglichen Schadensersatzes}

Inhalt und Höhe des Schadensersatzanspruchs blieben vorliegend weitgehend unerörtert, wenn auch die angesprochenen Fragen zur Konkurrenz zwi-

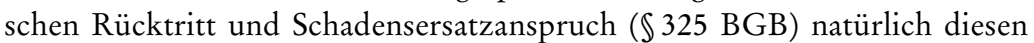
Fragenkomplex durchaus tangieren. Davon abgesehen hat sich bei Inhalt und Höhe kategorial nichts geändert, deshalb blieben sie unerörtert. Eine einzige Ausnahme ist freilich zu machen.

\section{Allgemeine Öffnung für immateriellen Schadensersatz ( $\iint 253 A b s .2,284 B G B$ )}

$\mathrm{Zu}$ finden ist sie in $\$ 284 \mathrm{BGB}$ und ab dem 1.8.2002 zusätzlich in $\ 253$ Abs. 2 BGB. Canaris listet $\$ 284$ BGB unter den „kleinen Abweichungen“ vom alten BGB auf, ${ }^{76}$ weder Münchener Kommentar noch Palandt benennen auch nur eine Literaturstelle, die sich im Schwerpunkt mit $\$ 284$ BGB auseinandersetzt. Jeweils geht es um die Liquidierbarkeit immateriellen Schadens. Vor dem 1.1.2002 war diese ein Thema allein beim deliktischen Schadensersatzanspruch, seitdem ist sie auch eines beim vertraglichen Schadensersatzanspruch. Und die zu beschreibende Tendenz gilt für beide genannten Normen.

a) Immaterieller Schaden auch im Vertrags- und allgemein im Schuldrecht (\253 Abs. $2 B G B$ )

Ungleich intensiver diskutiert wurde $\$ 253$ Abs. 2 BGB, ${ }^{77}$ neu im BGB mit Wirkung vom 1.8.2002. Die Norm ist wichtig, weil mit ihr ein Anspruch auf

76 Canaris, JZ 2001, 499, 522. Diese Norm geht zurück auf $\$ 327$ Abs. 1 S. 2 des Entwurfs der Schuldrechtskommission, vgl. Bundesminister der Justiz (Fn. 12), 173 f., 293; zur durchaus erheblichen Bedeutung im Grundsatz ausführlich Canaris, Äquivalenzvermutung und Äquivalenzstörung im Leistungsstörungsrecht des BGB, FS Wiedemann 2002, 3, 3 f. und 26-33.

77 Wagner, Das Zweite Schadensersatzrechtsänderungsgesetz, NJW 2002, 2049, 2053-2057; MünchKommBGB/Oetker, $\$ 253$ Rn. 10 ff. 
Ersatz immateriellen Schadens allgemein im Vertragsrecht, im Schuldrecht AT installiert wird, ja sogar allgemein im Schuldrecht, also insbesondere auch für die Gefährdungshaftung. ${ }^{78}$ Umgekehrt ist das Gewicht der Norm jedoch auch wieder begrenzt, weil letztlich nur die Konturen des deliktischen $\$ 847$ BGB a.F. übernommen wurden, sich die bisherige Rechtslage also im wesentlichen nur insoweit ändert, als Gehilfenverhalten ohne Exkulpation (nach $\$ 278$ BGB) zugerechnet wird und die vertragsrechtliche Beweislastumkehr für das Verschulden gilt ( $\$ 280$ Abs. 1 S. 2 BGB). Allerdings wagte der Gesetzgeber zum 1.8.2002 nicht einmal mehr, die zentrale Rechtsprechungsfortentwicklung - zum Persönlichkeitsrecht - nachzuzeichnen. ${ }^{79}$

\section{b) Ersatz immateriellen Schadens auch obne Verletzung der Person des Anspruchsstellers (etwa $\int 284 B G B$ )}

Schon mit Wirkung ab dem 1.1.2002 galt $₫ 284$ BGB. Praktisch ist die Norm in der Tat wohl nicht allzu bedeutend, es wurde im wesentlichen nur die BGH-Entscheidung im Stadthallenfall korrigiert. ${ }^{80}$ Hatte der Gläubiger Aufwendungen gemacht, hatte die Rechtsprechung zwar eine Rentabilitätsvermutung dahingehend aufgestellt, dass die Aufwendungen in jedem Fall bei Vertragsdurchführung auch erwirtschaftet worden wären, also die vergeblichen Aufwendungen den Mindestschaden darstellen. Dabei handelte es sich jedoch nur um eine Vermutung, und diese war insbesondere widerlegt, wenn die Aufwendungen einem ideellen Zweck dienten, etwa, wie im Stadthallenfall, der Durchführung einer Parteiversammlung. Aufwendungen kann der Gläubiger seit dem 1.1.2002 auch in diesem Fall verlangen.

Die Norm hat bei Gegnern wie Freunden der Reform ungewöhnlich viel Kritik erfahren. ${ }^{81}$ Das ist im Hinblick auf die geringe praktische Bedeutung zunächst einmal verwunderlich - in diesem Sinne handelt es sich im Sinne

${ }^{78}$ BT-Drucks. 14/7752, S. 14-16, 24 f.; Kropholler (Fn. 34), $\$ 253$ Rn. 2; MünchKommBGB/Oetker $\$ 253$ Rn. 18 (mit Auflistung entsprechender Klarstellungen in den Einzelnormen).

79 Maßgeblich waren BGHZ 26, 349 (Herrenreiter); 39, 124 (Fernsehansagerin). Befürchtet wurde eine Signalwirkung zu Lasten der Pressefreiheit. Vgl. (nicht so explizit) BT-Drucks. 14/7752, S. 24 f., 49 f. Deshalb ist nur die bisherige Rechtsprechung (mit der Einschränkung, dass Schmerzensgeld nur ultima ratio ist) anzuwenden: Kropboller (Fn. 34), $\$ 823$ Rn. 17; sowie, wenn auch etwas missverständlich, MünchKommBGB/Oetker $\$ 253$ Rn. 27.

${ }^{80}$ BGHZ 99, 182, bes. 198.

81 Einerseits Altmeppen, Untaugliche Regeln zum Vertrauensschaden und Erfüllungsinteresse im Schuldrechtsmodernisierungsentwurf, DB 2001, 1399, 1402-1405; Anwaltkommentar/Dauner-Lieb, $\mathbb{} 284 \mathrm{Rn}$. 12; andererseits aber auch etwa Schlechtriem (Fn. 16), Rn. 377; Stoll, Notizen zur Neuordnung des Rechts der Leistungsstörungen, JZ 2001, 589, 595 f.; positiv etwa Medicus (Fn. 16), Rn. 389. 
Canaris, der zur Verabschiedung maßgeblich beitrug, in der Tat nur um eine „kleine Abweichung“. Umgekehrt erscheint das Gewicht der Norm aus rechtsvergleichender und auch europarechtlicher Perspektive jedoch durchaus nicht unerheblich - sie könnte für eine Trendwende stehen. Aus dieser Perspektive erscheint die genannte Kritik dann jedoch zusätzlich auch noch als ein Beharren auf einer zunehmend vereinzelten nationalen Position, über die der internationale Trend hinweggeht. Canaris ist also auch darin zuzustimmen, dass die Norm ebenfalls grundsätzlichen Charakter hat und in die richtige Richtung weist.

\section{2. Öffnung auch rechtsvergleichend und europarechtlich nabeliegend}

In der Tat ist $\$ 284 \mathrm{BGB}$ - trotz geringer praktischer Bedeutung - in der Stoßrichtung wichtig, in mancher Hinsicht sogar wichtiger als $\ 253$ Abs. 2 BGB. Auf drei Aspekte ist hinzuweisen.

Mit $\$ 284$ BGB werden immaterielle Schäden auch außerhalb von Sachverhaltsgestaltungen unter Rechtsschutz gestellt, wie sie bisher $\$ 847$ BGB a.F. umschrieb (und heute noch immer $\$ 253$ Abs. 2 BGB). Diese sind dadurch gekennzeichnet, dass es zu einem Eingriff in die Person (Körper, Leben, Persönlichkeit) gekommen sein muss und zwar in die Person des Anspruchsstellers selbst. Die gegenteilige Position, die weitergehend immateriellen Schaden liquidiert sehen wollte, wurde immer wieder mit dem Argument abgelehnt, dass immaterielle Schäden schwer zu kalkulieren seien. ${ }^{82}$ Im Ergebnis führte dies freilich zu einer - im 19. Jahrhundert teils auch in anderen Punkten zu konstatierenden - Höherbewertung materieller Werte gegenüber immateriellen. Freilich hängt dies keineswegs notwendig, wie teils behauptet, mit den Beginnen der Wohlfahrtsökonomie zusammen, hatte doch gerade Bentham im Ausgangspunkt die höchste Summe des Glücks, nicht nur der materiellen Werte als Ziel postuliert. Jedenfalls erfolgte 2002 eine Korrektur: Rechtsvergleichend verkörperte das deutsche Recht eine Extremposition - nicht nur verglichen mit dem U.S.-amerikanischen Recht, das teils als exzessiv empfunden wird. Auch etwa in Frankreich werden trotz offenem Deliktstatbestand (Art. 1382 Code Civil), also obwohl auf der Tatbestandsseite die Kanalisierung sogar geringer ist, ${ }^{83}$ ungleich mehr Fallgruppen immaterieller Schäden liquidiert. Es findet sich eine ausziselierte Fallgruppenbildung, liquidiert werden etwa: „préjudice de souffrance“, „préjudice d'agrément“, „préjudice

82 Vgl. dazu und zu anderen Argumenten gegen eine Ausweitung des Ersatzes immaterieller Schäden Lange, Schadensersatz ${ }^{2}, 1990$, \$ 7 I 1 (S. 425-427).

83 Die Norm lautet: „Tout fait quelconque de l'homme qui cause à autrui un dommage oblige celui par la faute duquel il est arrivé à le réparer.“ 
sexuel“, „préjudice de contamination“, „préjudice d'affection“. ${ }^{84}$ Auch das englische common law ist ungleich weniger restriktiv. ${ }^{85}$ Natürlich ist die Berechnung immaterieller Schäden schwierig und ist letztlich bis zu einem gewissen Grad auch eine dezisionistische Bewertung nötig. Die Schäden jedoch unter Hinweis auf diese Schwierigkeiten pauschal unliquidiert zu lassen, heißt das Kind mit dem Bade ausschütten. Anders als etwa bei $\$ 831$ BGB wurde bei diesem recht zentralen Manko des deutschen Delikts- und allgemein Schadensrechts wenig kuriert. Die Korrektur erfolgt jetzt zweifach: Es kommen anders geartete Fallgruppen hinzu ( $\$ 284 \mathrm{BGB})$, und liquidierbar sind immaterielle Schäden jetzt auch nicht mehr nur im Deliktsrecht, sondern in weiteren Rechtsgebieten ( $\$ 253$ Abs. 2 BGB).

Ein zweiter Punkt betrifft die Rückwirkung auf die deutsche Rechtsprechung. Denn wenn die deutsche Rechtsprechung eine Rechtsfortbildung bisher außerhalb vergleichbar persönlichkeitsbezogener Fälle abgelehnt hat und auf den Gesetzgeber verwies, mag sich ebendies nunmehr geändert haben. Denn der Gesetzgeber eröffnete jetzt zwei neue Fallgruppen. Immerhin war ja auch vorher noch $\ 651 \mathrm{f}$ Abs. 2 BGB (die entgangene Urlaubsfreude) ins Gesetz gekommen. Die gesetzgeberische Tendenz geht also, wenn immer der Gesetzgeber tätig wurde, jeweils dahin, die Ersatzfähigkeit des immateriellen Schadens zu bejahen. Daher liegt es nahe, dass etwa in einem Fall von Geschlechtsdiskriminierung a maiore immaterieller Schaden ersetzt werden muss, wenn schon die - vergleichsweise banale - entgangene Urlaubsfreude ersatzfähig ist.

Mit dem Stichwort entgangene Urlaubsfreude ist ein dritter Aspekt angesprochen, der die für möglich gehaltene Tendenz befördern wird. Nicht zuletzt ist auch im Europäischen Privatrecht mit dem Gebot effektiver Sanktionen ${ }^{86}$ in Fragen der Liquidierbarkeit immaterieller Schäden eher eine breite Fallgruppenbildung zu erwarten als ein weitgehender Ausschluss nach dem Modell des bisherigen deutschen Rechts. Da die Frage der Geschlechtsdiskriminierung im Zentrum europavertragsrechtlicher Politik steht, ${ }^{87}$ gilt das ge-

${ }^{84}$ Das umfasst etwa AIDS, auch die Trauer von Angehörigen und (bei Beweislastumkehr) sogar Lebensgefährten, Mobbing u.a. Vgl. zu all dem etwa Viney/Jourdain, Traité de Droit Civil², 1998, 36-53. Rechtsvergleichend knapp Anmerkung 4 zu Art. 9: 501 der European Principles of Contract Law (Fn. 50).

${ }^{85}$ Deakin/Johnston/Markesinis, Markesinis and Deakin's Tort Law, 5. Aufl. 2003, 827-831. Hauptgruppen sind: Pain and suffering (mit mental distress), loss of amenities und damages for bereavement (Lebenserwartung).

${ }^{86}$ Sie müssen „jedenfalls wirksam, verhältnismäßig und abschreckend“ sein: EuGH 21.9.1989 - Rs. 68/88 Kommission/Griechenland, Slg. 1989, 2965, 2985; 10.7. 1990 - Rs. C-326/88 Hansen, Slg. 1990, I-2911, 2935.

87 Vgl. nur Art. 119 EG; Richtlinie 75/117/EWG des Rates vom 10.2.1975 zur Angleichung der Rechtsvorschriften der Mitgliedstaaten über die Anwendung des Grund- 
rade auch für diesen Bereich. Voraussichtlich wird sich die EuGH-Rechtsprechung stützen auf: die Produkthaftungs-Richtlinie und auch die Staatshaftungs-Rechtsprechung, letztlich aber jede Europäisch angeordnete Primärpflicht, deren Sanktionierung der Europäische Gesetzgeber in die Hände der Mitgliedstaaten legt. Das Urteil in Sachen Simone Leitner zeigt, dass der EuGH die Ersatzfähigkeit immaterieller Schäden auch auf der Grundlage von Normen fordert - hier Art. 5 der Pauschalreise-Richtlinie-, die nur von Schadensersatz oder gar nur von effektiver Durchsetzung ganz allgemein sprechen. ${ }^{88}$

\section{Resümee und Ausblick}

Die Schuldrechtsmodernisierung führte zu einem Umbruch im System des Leistungsstörungsrechts. Nicht mehr die Unterscheidung nach Verstoßtatbeständen steht im Vordergrund, sondern die Unterscheidung nach Rechtsfolgen. Letztere stehen auch klarer gegeneinander. Dies gilt keineswegs nur, jedoch insbesondere für den Schadensersatz aus Vertrag.

In der Tat folgen die klassisch unterschiedenen Verstoßtatbestände beim Schadensersatz, jedoch auch sonst im Leistungsstörungsrecht, d.h. in Fragen des Wegfalls der Primärleistungspflicht und des Rücktritts, einem einheitlichen Regime. Kein Sonderregime gilt zunächst für die Unmöglichkeit. Sie ist unzweifelhaft irrelevant für die Frage nach dem Schadensersatz statt der Leis-

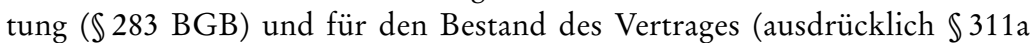
Abs. 1 BGB), richtigerweise auch für den Wegfall der Primärleistungspflicht $(\$ 275$ BGB) und für die Eröffnung einer zweiten Ebene des Vertretenmüssens (Kennenmüssen eines von außen herangetragenen, unbeherrschbaren Hindernisses, $\mathbb{3} 311 \mathrm{a}$ Abs.2). Ebenfalls dem allgemeinen Regime folgt die Schlechterfüllung, sowohl beim Schadensersatz neben der Leistung ( $\mathbb{2 8 0}$ Abs. 1 BGB) als auch beim Schadensersatz statt der Leistung ( $\$ 281$ BGB). Dass der Gesetzgeber Letzteres noch in den letzten Wochen des Verfahrens

satzes des gleichen Entgelts für Männer und Frauen, ABl.EG 1975 L 45/19; Richtlinie 76/207/EWG des Rates vom 9.2.1976 zur Verwirklichung des Grundsatzes der Gleichbehandlung von Männern und Frauen hinsichtlich des Zugangs zur Beschäftigung, zur Berufsbildung und zum beruflichen Aufstieg sowie in bezug auf die Arbeitsbedingungen, ABl.EG 1976 L 39/40; Richtlinie 97/80/EG des Rates vom 15.12.1997 über die Beweislast bei Diskriminierung aufgrund des Geschlechts, ABl.EG 1998 L 14/6; und aus der Literatur etwa Grundmann, Europäisches Schuldvertragsrecht (Fn. 9), 3.10, 3.11, allein die Monographien erreichen nahezu eine dreistellige Zahl, vgl. dort und Standardkommentare zu Art. 119 EG und \$611a BGB.

${ }^{88}$ EuGH 12.3.2002 - Rs. C-168/00 Simone Leitner/TUI, Slg. 2002, I-2631; zur Richtlinie 90/314/EWG des Rates vom 13.06.1990 über Pauschalreisen, ABl.EG 1990 L $158 / 59$. 
sicherstellte, ist ein erheblicher Fortschritt gegenüber den Vorschlägen der Leistungsstörungskommission (2001). Doch auch $\$ 275$ BGB gilt für die Schlechterfüllung und natürlich bildet die bereits anfänglich feststehende Unmöglichkeit, fehlerfrei zu leisten, kein Wirksamkeitshindernis. Allein der Verzug folgt - für den Schadensersatz neben der Leistung, nicht jedoch für den Schadensersatz statt der Leistung und den Rücktritt - Sonderregeln: Anders als in $₫ 280$ Abs. 1 BGB werden nicht nur Pflichtverstoß und Vertretenmüssen, sondern zusätzlich Mahnung vorausgesetzt - meist jedoch nur formal: Nimmt man nämlich die (seit 2002 weiter umrissenen) Ausnahmen vom Mahnerfordernis hinzu, so wird klar, dass für den Vertragsgestalter, der lege artis arbeitet, der Verzugsschaden (als Schadensersatz neben der Leistung) ebenfalls durchweg ohne Mahnung eröffnet ist, also nach dem Regime, das bei allen sonstigen Pflichtverstößen gilt.

Wichtig auf Tatbestandsseite ist nur die Unterscheidung nach leistungsbezogenen Pflichten und nicht leistungsbezogenen Pflichten. Deshalb ist nicht nur die Unmöglichkeit als Kategorie unwichtig für den Schadensersatz, sondern auch die Schlechterfüllung. Jeweils geht es um leistungsbezogene Pflichten. Alle leistungsbezogenen Pflichtverstöße unterfallen $\$ 280$ BGB (beim Schadensersatz neben der Leistung), alle auch $\$ 281$ BGB (beim Schadensersatz neben der Leistung) und alle berechtigen zum Rücktritt, also (auch) zum Schadensersatz neben den beiden zurückabgewickelten Leistungen. Abgetrennt wird nicht die Schlechterfüllung, sondern allein die Verletzung nicht leistungsbezogener Pflichten, in $\$ 282$ BGB im Gegensatz zu $\$ 281$ BGB und entsprechend im $\ 324$ BGB im Gegensatz zu $\ 323$ BGB. Abgetrennt wird ansonsten nur noch die Teilleistung, mit anderen Worten: Soweit das ohne Eingriff in die Interessen des Gläubigers möglich ist, wird der Vertrag de facto aufgespalten, um den Eingriff in Schuldnerinteressen möglichst gering zu halten.

Dass die Unterscheidung nach Verstoßtatbeständen 2001 gesetzgebungstechnisch wieder „rehabilitiert“ wurde (Canaris), also ein System der Rechtsfolgendifferenzierung kombiniert wurde mit einem System der Differenzierung nach Verstoßtatbeständen („mittlere Ebene“), erscheint mir als ein Rückschritt gegenüber den Vorschlägen der Schuldrechtskommission. Es werden Normen ausgebildet und nebeneinander gestellt, die den Anschein erwecken, in Rechtsfolge oder Tatbestand könnten Unterschiede bestehen, obwohl solche in Wirklichkeit gänzlich fehlen (etwa bei $\$ 283 \mathrm{BGB}){ }^{89} \mathrm{Zu}$ gleich werden allgemeine Gedanken nicht als solche ausgebildet (etwa im Fal-

${ }^{89}$ Ungleich einfacher gestaltet wären $\$ \$ 281-283$ BGB in einer einzigen - inhaltlich allein die heutige Rechtslage reflektierenden - Norm mit folgendem Wortlaut (zu Abs. 2 vgl. auch Text nach Fn. 35): 


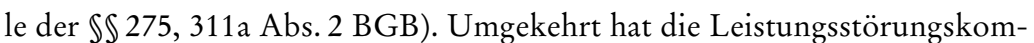
mission (2001) jedoch in einer Reihe einzelner Normen Einzeltatbestandsmerkmale klarer herausgearbeitet, etwa in $\$ 275$ Abs. 2 BGB („grobes Missverhältnis“ und Gläubigerinteresse als Bezugspunkt) oder mit $\$ 311$ a Abs. 2 BGB. Wäre die Arbeit auf Letzteres beschränkt geblieben, wäre der Systemwechsel klarer hervorgetreten und wären damit auch die Stärken des deutschen Rechts einfacher zu transportieren gewesen. So ist das deutsche Recht etwa den European Principles darin überlegen, dass der Verpflichtete, wenn seine Primärleistung zurückgewiesen werden soll, durch Fristsetzung durchgängig eine „zweite Chance“ erhalten soll. Die Arbeit der Leistungsstörungskommission (2001) wäre dann uneingeschränkt positiv zu bewerten gewesen, Leistungsstörungskommission (2001) und Schuldrechtskommission hätten sich (noch) besser ergänzt.

Viel wichtiger als die (regelmäßig nur formale) Differenzierung zwischen Verstoßtatbeständen ist der Schnitt zwischen den Rechtsfolgen. Denn nach Rechtsfolgen, (fast) nicht mehr nach Tatbeständen wird differenziert. Die abweichende Gesetzgebungstechnik, die noch die Wichtigkeit der Differenzierung nach Verstoßformen suggeriert, führt insoweit in die Irre. Zum Übergang von einem verstoßtatbestandsorientierten System zu einem rechtsfolgenorientierten System kommt ein Zweites hinzu: Auch innerhalb der Rechtsfolgen, bei der hier zu erkennenden Differenzierung, ist nochmals grundsätzlich umzudenken. Wohl nicht der Schnitt zwischen Schadensersatz einerseits und Rücktritt andererseits ist der praktisch wichtigste, wie dies die

\$281 Schadensersatz statt der Leistung

(1) Soweit der Schuldner gegen Vertragspflichten nach $\$ 241$ verstößt, kann der Gläubiger unter den Voraussetzungen des $\$ 280$ Abs. 1 Schadensersatz statt der Leistung verlangen, wenn er dem Schuldner eine angemessene Frist zur Leistung oder Nacherfüllung bestimmt hat. Kommt nach Art der Pflichtverletzung eine Fristsetzung nicht in Betracht, so tritt an deren Stelle eine Abmahnung.

(2) Eine Fristsetzung oder Abmahnung ist entbehrlich, wenn der Schuldner die Erfüllung der vertraglichen Pflicht ernsthaft und endgültig verweigert oder wenn besondere Umstände vorliegen, die unter Abwägung der beiderseitigen Interessen die sofortige Geltendmachung des Schadensersatzanspruchs rechtfertigen.

(3) Der Gläubiger kann Schadensersatz statt der Leistung nicht verlangen, wenn die Pflichtverletzung unerheblich ist. Außerdem kann er, wenn der Schuldner einen Teil leistet, Schadensersatz statt der ganzen Leistung nur verlangen, wenn er an der Teilleistung kein Interesse hat, und, wenn der Schuldner allein gegen eine nicht leistungsbezogene Nebenpflicht verstößt, nur, wenn ihm eine Leistung durch den Schuldner nicht mehr zuzumuten ist.

(4) Der Anspruch auf die Leistung ist ausgeschlossen, sobald der Gläubiger statt der Leistung Schadensersatz verlangt hat.

(5) Verlangt der Gläubiger Schadensersatz statt der Leistung, so ist der Schuldner

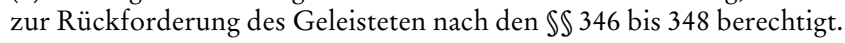


herkömmliche Dogmatik nahelegen würde. Der Unterschied zwischen Rechtsfolgen bei Vertretenmüssen und Rechtsfolgen ohne gesonderte Prüfung des Vertretenmüssens ist eher gering, zumal wenn der Gedanke des \$311a Abs. 2 BGB, wie hier vorgeschlagen, verallgemeinert wird. Er wird auch in Europa, wo die Garantiehaftung jedenfalls im Vertragsrecht dominiert, wohl kaum mitgegangen werden. Wichtiger erscheint regelmäßig - auch schon in Deutschland! - der Schnitt zwischen den Rechtsfolgen, die die Primärleistung des Verpflichteten intakt lassen (Schadensersatz neben der Leistung), und denen, bei denen dies nicht der Fall ist (Rücktritt und Schadensersatz neben der Leistung). Diesen Schnitt bezeichnet auch das regelmäßig praktisch wichtigere Zusatzerfordernis (neben Pflichtverstoß), das Fristsetzungserfordernis, das für den Verpflichteten der Eröffnung einer zweiten Chance gleich kommt. Rücktritt und Schadensersatz neben der Leistung sind daher in den Voraussetzungen (abgesehen vom Vertretenmüssen) auch praktisch identisch ausgestaltet.

Dieses System, so scheint es mir, kann im Europäischen Diskurs durchaus bestehen. Noch leichter wäre dies gewesen, wäre dieses System noch eindeutiger hervorgetreten. 